\title{
MILLENNIAL MESSAGES FOR QCD FROM THE SUPERWORLD AND FROM THE STRING
}

\author{
M.J. STRASSLER \\ Department of Physics, University of Washington, P.O. Box 351560, Seattle WA \\ 98195-1560 USA
}

\begin{abstract}
Supersymmetric gauge theories have had a significant impact on our understanding of QCD and of field theory in general. The phases of $\mathcal{N}=1$ supersymmetric QCD (SQCD) are discussed, and the possibility of similar phases in non-supersymmetric QCD is emphasized. It is described how duality in SQCD links many previously known duality transformations that were thought to be distinct, including OliveMontonen duality of $\mathcal{N}=4$ supersymmetric gauge theory and quark-hadron duality in (S)QCD. A connection between Olive-Montonen duality and the confining strings of Yang-Mills theory is explained, in which a picture of confinement via nonabelian monopole condensation — a generalized dual Meissner effect — emerges explicitly. Similarities between supersymmetric and ordinary QCD are discussed, as is a non-supersymmetric QCD-like "orbifold" of $\mathcal{N}=1$ Yang-Mills theory. I briefly discuss the recent discovery that gauge theories and string theories are more deeply connected than ever previously realized. Specific questions for lattice gauge theorists to consider are raised in the context of the first two topics. (Published in "At the Frontier of Particle Physics: Handbook of QCD", M. Shifman, editor)
\end{abstract}

\section{Introduction: Beyond QCD}

The field of supersymmetric gauge theory is fascinating, technical, and vast, and obviously cannot be reviewed in a single article. My limited intention is to give a qualitative and conceptual overview, with an eye toward conveying what I see as some of the most important messages of recent advances for our understanding of QCD. Consequently, this article will consist largely of results that I see as relevant to QCD; only rarely will it present the evidence for these results or many technical details, and referencing will be limited. The interested reader is directed to more focussed reviews on the superworld., ${ }^{1,2,3}$

The purpose of my writing such a review? This is perhaps conveyed best by the words of Tom DeGrand, when he once asked me to speak to an audience of lattice gauge theorists: he requested that I encourage them "to change the line in their code that sets $N_{c}, N_{f}=3$." I hope to convince you that a systematic exploration of theories other than real-world QCD is an important and exciting direction for research, and that placing QCD in the context of a wider variety of theories may become a powerful tool for understanding it.

There are several reasons why physicists should seek a detailed understanding of gauge theories beyond real-world QCD. First, we can use them to gain insight into the properties of the real world. It would be helpful to know which 
aspects of the strong interactions are special to $N_{c}=N_{f}=3$ and which ones are generic, or at least common to many models. Second, a theory with behavior only vaguely similar to QCD may be responsible for electroweak symmetry breaking, as in technicolor and topcolor models. Examples of non-QCD-like theories are the fixed-point models which have been discussed recently. ${ }^{5}$ Third, it is important to test numerically some of the analytic predictions of supersymmetric gauge theory, in part to close some remaining loopholes in the analytic arguments. In addition, there are possible applications to condensed matter.

Four-dimensional supersymmetric gauge theories are good toy models for non-supersymmetric QCD and its extensions. Some of these theories display confinement; of these, some break chiral symmetry but others do not.6,7 The mechanism of confinement occasionally can be understood using a weaklycoupled "dual description" $8,9,10$ (an alternate set of variables for describing the same physics.) However, what is more striking is that most of these theories do not confine! ${ }^{10}$ Instead, their infrared physics is governed by other, unfamiliar phases, often described most easily using dual variables. (Here and throughout, "phase" refers to the properties of the far infrared physics at zero temperature.) The phase diagram, as a function of the gauge group, matter content, and interactions, is complex and intricate. ${ }^{10}$

I will show that these theories have important connections with nonsupersymmetric theories. While direct applications to QCD are few, there are nonetheless important qualitative insights which can be gained. Explicitly broken $\mathcal{N}=4$ and $\mathcal{N}=2$ supersymmetric Yang-Mills (SYM) theories give windows into confinement. Chiral symmetry breaking can be explored in $\mathcal{N}=1 \mathrm{SYM}$ and in supersymmetric QCD (SQCD). The large- $N_{c}$ limit offers the connection between an ordinary field theory and a string theory on a space of negative curvature, ${ }^{11,12,13,14}$ and an "orbifold conjecture" relating a non-supersymmetric QCD-like theory to $\mathcal{N}=1 \mathrm{SYM}$. I'll also make some comments about avenues that lattice QCD theorists might want to pursue.

\section{Pure $\mathcal{N}=1$ Super-Yang-Mills}

Let us begin with a discussion of the pure $\mathcal{N}=1$ supersymmetric Yang-Mills (SYM) theory - simply $S U(N)$ gauge theory with a vector boson (gluon) $A_{\mu}$ and a Majorana spinor (gluino) $\lambda_{\alpha}$, and Lagrangian

$$
\mathcal{L}=\frac{1}{2 g^{2}}\left[\operatorname{tr} F_{\mu \nu} F^{\mu \nu}+i \bar{\lambda} \not D \lambda\right]
$$

Quantum mechanically, SYM bears some resemblance both to ordinary nonsupersymmetric YM and QCD, as it exhibits both confinement and chiral symmetry breaking. 


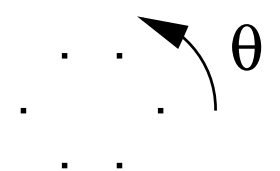

Figure 1: The vacua of SYM theory are rotated by the theta angle.

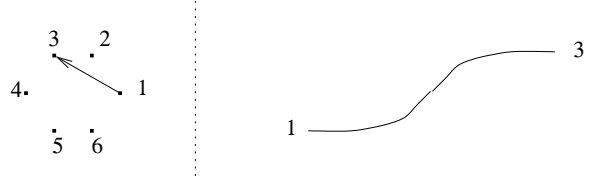

Figure 2: Domain walls can interpolate between vacua of SYM theory.

Let us examine a bit more closely the issue of chiral symmetry breaking. The classical $U(1)$ axial symmetry $\lambda \rightarrow \lambda e^{i \sigma}$ is anomalous; since $\lambda$ has $2 N$ zero modes in the presence of an instanton, only a $\mathbf{Z}_{2 N}$, under which $\lambda \rightarrow \lambda e^{i \pi n / N}$, is anomaly-free. The dynamics causes a gluino condensate $\langle\lambda \lambda\rangle$ to form, breaking this discrete axial symmetry further to a $\mathbf{Z}_{2}$ under which $\lambda \rightarrow-\lambda$. This leads to $N$ equivalent vacua with $\langle\lambda \lambda\rangle=\Lambda^{3} e^{i(2 \pi n / N)}$. Note that an instanton in this theory comes with a factor $\Lambda^{3 N}$; the form of the gluino condensate suggests it is induced by fractional instantons, indeed, by $1 / N$ of an instanton.

If we shift the theta angle by $\theta \rightarrow \theta+\alpha$, then $\Lambda^{3} \rightarrow \Lambda^{3} e^{i \alpha / N}$, and the $N$ equivalent vacua (Fig. 1) are rotated by an angle $\alpha / N$. Any given vacuum only comes back to itself under $\theta \rightarrow \theta+2 \pi N$, but the physics is invariant under $\theta \rightarrow \theta+2 \pi$. For fixed $\theta$, the theory can have domain walls separating regions in different vacua; as in Fig. 2, the condensate $\langle\lambda \lambda\rangle$ can change continuously from $\Lambda^{3} e^{2 \pi i n / N}$ to $\Lambda^{3} e^{2 \pi i n^{\prime} / N}$.

Since $\mathcal{N}=1$ SYM confines, it exhibits electric flux tubes. As in ordinary YM, the confining "strings" carry charge in the center of the gauge group; for $S U(N)$ they carry quantum numbers $k$ in $\mathbf{Z}_{N}$. In later sections, I will discuss these flux tubes, their charges, and their tensions $T_{k}$ in much greater detail.

It is important to note that similar properties - equivalent discrete vacua, domain walls, confining strings, and possible fractional instantons - should appear (and should be searched for) in certain non-supersymmetric gauge theories with fermions. In fact, we expect them to occur in a set of models I will discuss in a later section - in so-called "orbifolds" of $\mathcal{N}=1$ SYM theory.

What happens if we break supersymmetry by adding a mass $m$ to the gaugino? 


$$
\mathcal{L} \rightarrow \mathcal{L}+m \lambda \lambda
$$

The degeneracy of the $N$ SYM vacua will be broken; which one has lowest energy will depend on the phase of $m$. As its phase is rotated, the preferred vacuum will shift from one to the next. All domain walls become unstable (except for special choices of $\arg (m)$ for which the two lowest vacua become degenerate.) However, the energy gap, the spectrum, and the general features of confinement will not be greatly altered if $|m| \ll|\Lambda|$.

An issue for study using lattice methods is whether there are any qualitative transitions in the physics of the theory as $m$ increases and pure YM is recovered. The question is whether the properties of SYM - for example, the physics leading to confinement - is relevant for understanding pure YM. Clearly, this rests in part on whether the two theories are continuously connected, or whether they are separated in parameter space by a phase transition. For example, consider the tensions $T_{k}$ of the flux tubes as a function of the mass $m$; do they change continuously, or do they show a jump at some value of $m$ ? It would also be interesting to understand the behavior of $\langle\lambda \lambda\rangle$ as a function of $m$.

Before returning to a study of confinement in SYM, I now want to turn to supersymmetric gauge theories with matter, and some of their remarkable properties.

\section{Gauge Theories with Matter}

Supersymmetric gauge theories teach us that the matter content of a theory plays an essential role in determining its basic physics. In particular, the phase at zero temperature of a gauge theory (that is, its properties in the far infrared) depends in a complicated way on (1) its gauge group $G$, (2) its matter representations $R$, and (3) the self-interactions $\mathcal{L}_{m}$ of the matter, including non-renormalizable interactions. Recent work has shown that the phase structure of $\mathcal{N}=1$ supersymmetric theories is complex and intricate, and it is reasonable to expect that the same will be true of non-supersymmetric theories.

Among the surprises discovered in the supersymmetric context are that there are new and unexpected phases unknown in nature or previously thought to be quite rare. It also appears that duality is a fundamental property of field theory (and also of gravity, and even between gravity and gauge theory!) Certain accepted or at least popular lore has been refuted as well: the beta function does not, by itself anyway, determine the phase of a gauge theory; 
confinement does not always cause chiral symmetry breaking; and fixed points in four dimensions are not at all rare - in fact, they are commonplace!

\subsection{Phases of $S Q C D$}

Supersymmetric QCD consists of $\mathcal{N}=1 S U(N)$ SYM with $N_{f}$ flavors of massless scalars (squarks) $Q$ and fermions (quarks) $\psi$ in the fundamental representation, along with anti-squarks and anti-quarks $\tilde{Q}$ and $\tilde{\psi}$ in the anti-fundamental representation. The Lagrangian is

$$
\mathcal{L}=\mathcal{L}_{S Y M}+|D Q|^{2}+i \bar{\psi} \not D \psi+|D \tilde{Q}|^{2}+i \overline{\tilde{\psi}} \not D \tilde{\psi}+\text { some interactions }
$$

Following the work of Seiberg ${ }^{10}$ and others ${ }^{15,16,17,18,19}$ we now know that SQCD has many phases. Fig. 3 shows the phase diagram of the infrared behavior of the theory as a function of $N$ and $N_{f}$, as determined by Seiberg. ${ }^{10}$ (Note that supersymmetric theories generally have continuous sets of inequivalent vacua, called "moduli spaces;" for each theory I have only listed the phase of the vacuum with the largest unbroken global symmetry. Also, unless otherwise specified, I am always considering the case of strictly massless matter.)

1) The Free Electric Phase: When $N_{f}>3 N$, the theory has a positive beta function and flows to weak coupling. The theory is free in the infrared in terms of the original variables.

2) The Non-Abelian Coulomb Phase: When $3 N>N_{f}>\frac{3}{2} N$, the theory is asymptotically free, but its beta function hits a zero at a finite value of the gauge coupling. The low-energy theory is an infrared fixed point, an interacting conformal field theory, which has no particle states. Its operators have nontrivial anomalous dimensions, some of which can be exactly computed. The theory has a "dual description" using "magnetic" variables: in these variables, the conformal theory is seen as an infrared fixed point of a different gauge theory, one with $S U\left(N_{f}-N\right)$ gauge group and $N_{f}$ flavors as well as $N_{f}^{2}$ gauge-neutral massless fields.

3) The Free Magnetic Phase: When $\frac{3}{2} N \geq N_{f} \geq N+2$, the theory flows to strong coupling in the infrared. The low-energy physics can be described in terms of composite fields, of spin $0, \frac{1}{2}$, and 1, many of which are nonpolynomial - indeed, non-local - in terms of the original fields. The theory describing these composites is again a gauge theory, with its own conceptually separate gauge symmetry! Specifically, the theory of these "dual" fields is an $S U\left(N_{f}-N\right)$ gauge theory with $N_{f}$ flavors and $N_{f}^{2}$ neutral massless fields. This dual theory has a positive beta function, and so the magnetic degrees of freedom are infrared-free. Thus, while the theory is strongly coupled in 


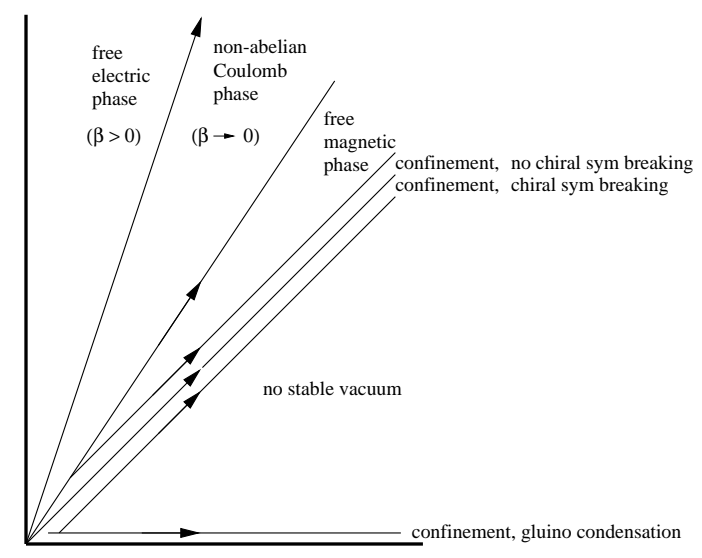

Figure 3: The phases of SQCD, as a function of $N_{f}$ (vertical axis) and $N$ (horizontal axis.)

terms of the original variables, these magnetic "quasi-particles" give a good perturbative description of the low-energy physics.

4) Confinement Without Chiral Symmetry Breaking: For $N_{f}=N+1$, the theory confines. In this case the low energy theory is an infrared-free linear sigma model, describing composite mesons and baryons (of spin 0 and $\frac{1}{2}$ ) that are polynomial in the original fields.

5) Confinement With Chiral Symmetry Breaking: For $N_{f}=N$, the theory again confines. Again the light particles are massless mesons and (scalar) baryons, but now the theory describing them breaks chiral symmetry and becomes a non-linear sigma model.

6) $0<N_{f}<N$ : When $N_{f}=N-1$, instantons generate an unstable potential for the squarks; a similar effect, due to gaugino condensation (or perhaps fractional instantons?) occurs for $N-2 \geq N_{f} \geq 1$. There is no stable vacuum if the quarks and squarks are massless.

For $N_{f}=0$, we have pure $\mathcal{N}=1 \mathrm{SYM}$, which is in phase (5).

As another example, consider $S O(N)$ with $N_{f}$ flavors in the vector representation, whose dual is $S O\left(N_{f}-N+4\right){ }^{10,15}$ In this case we have phase (1), $N_{f} \geq 3(N-2)$; phase (2), $3(N-2)>N_{f}>\frac{3}{2}(N-2)$; phase (3), $\frac{3}{2}(N-2) \geq N_{f}>N-3$; phase (4), $N_{f}=N-3, N-4$. As before $N_{f}<N-4$ has no vacuum, except $N_{f}=0$ which has vacua with phase (5).

I will now elaborate on these phases, and illustrate the dependence of the phase structure on the details of the theory. 

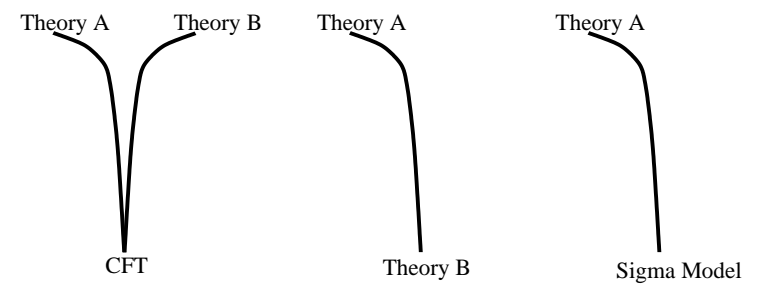

Figure 4: (a) Non-abelian Coulomb Phase: two gauge theories flow to the same conformal fixed point. (b) Free Magnetic Phase: gauge theory A becomes strongly coupled, and at low-energy mysteriously turns into gauge theory B. (c) Confining Phase: theory A becomes strongly coupled, and mysteriously turns into a theory of mesons and baryons.

\subsection{Non-Abelian Coulomb Phase}

This phase can be seen in perturbation theory, both in QCD and SQCD, when the number of colors and flavors is large and the one-loop beta function is extremely small by comparison; such fixed points are often called Banks-Zaks fixed points, ${ }^{20}$ though they were discussed by earlier authors as well. What is new here is that this phase exists far beyond perturbation theory into an unexpectedly wide range of theories. Furthermore, these fixed points exhibit duality: there exist multiple gauge theories which flow to the same conformal field theory, as illustrated schematically in Fig. 4a. Thus, there are multiple sets of variables by which the conformal field theory may be described. This is analogous to Olive-Montonen duality in $\mathcal{N}=4 \mathrm{SYM}$, which is a conformal field theory; more on this later.

\subsection{Free Magnetic Phase}

This spectacular phase was entirely unexpected. Here, the theory flows to strong coupling, and its infrared physics is described by weakly coupled composite matter and gauge fields. These composites are non-local with respect to the original degrees of freedom, and have an unrelated gauge symmetry. The duality transformation which acts on the infrared fixed points of the nonabelian Coulomb phase operates in the free magnetic phase by exchanging the fundamental theory of the ultraviolet with the infrared-free composite theory, as illustrated in Fig. 4b. Note that the free magnetic phase is not a confining phase. ${ }^{21,22,23}$. 


\subsection{Confinement with and without chiral symmetry breaking}

In the confining theories, the low-energy description is a theory of gauge singlets built from polynomials in the original degrees of freedom. The main difference between chiral-symmetry-preserving and -breaking theories is in the interaction Lagrangian, which determines the symmetries preserved by the vacuum.

As in the free magnetic phase, the duality transformation exchanges the ultraviolet theory with the infrared one - the quarks and gluons of the gauge theory are exchanged with the mesons and baryons of the linear or non-linear sigma model (Fig. 4c). At this point duality begins to resemble what is done in real-world QCD when we rewrite the theory in term of hadrons and the chiral Lagrangian. This strongly suggests that the QCD/chiral-Lagrangian "duality" transformation is conceptually related to electromagnetic duality, its generalization to Olive-Montonen duality, and its lower-dimensional cousins. I will make this more precise in later sections.

Note that the word "confinement" has been used loosely above, and it is essential to make an important distinction. The cases $S U(N)$ with $N_{f}=$ $N+1, N$ are examples of "complementarity", where the confining and Higgs phases are actually two regions in a single phase. ${ }^{24}$ There is no Wilson loop with an area law; all sources can be screened by the massless fields, and so no confining string can form. By contrast, a spinor-valued Wilson loop detects the confinement in $S O$ theories with vectors, while Wilson loops in, for example, the $\mathbf{N}$ representation, can detect the confinement in the pure $S U(N)$ and $S O(N)$ SYM. In these cases the notion of confinement is precise.

\subsection{Dependence of Phase on Gauge Group and Matter Content}

While the phase structures of different gauge theories roughly resemble one another, they are not by any means identical. Consider, for example, $S O(8)$ gauge theory with six fields in the vector representation. This theory is in the free magnetic phase, with an abelian dual gauge group. ${ }^{15}$ If we instead take $S O(8)$ with five vectors and one spinor - this theory has the same one-loop beta function as the previous one - then the theory is confining, and has a vacuum which does not break its chiral symmetries. As another example, compare $S U(N)$ SQCD with $N_{f}=N$ to $\mathcal{N}=2 S U(N)$ SYM. The two theories have the same beta function, but the first is in the confining phase with chiral symmetry breaking, while the second is in the free magnetic phase. 


\subsection{Dependence of Phase on Interactions}

The phase of a theory also depends strongly on the interactions between the matter fields. For example, consider $S U(4)$ SQCD with $N_{f}$ flavors of squarks $Q_{i}$, anti-squarks $\tilde{Q}^{i}$, quarks and anti-quarks $\psi_{i}, \tilde{\psi}^{i}$. Consider taking a "superpotential" $W=y Q_{1} Q_{2} Q_{3} Q_{4}, y$ a coupling with dimensions of inverse mass; this adds the following perturbation to the Lagrangian:

$$
\left[\sum_{i, j} \frac{\partial^{2} W}{\partial Q_{i} \partial Q_{j}} \psi_{i} \psi_{j}+\text { h.c. }+\sum_{i}\left|\frac{\partial W}{\partial Q_{i}}\right|^{2}\right] .
$$

Although this perturbation is irrelevant at weak coupling, it can be important in the infrared, depending on $N_{f}$. In particular,

- For $N_{f}=8$, the theory is in the non-abelian coulomb phase: the perturbation (3) is irrelevant, as in the classical limit.

- For $N_{f}=7$, the theory without the perturbation is in the non-abelian coulomb phase, but the perturbation is relevant and drives the theory to a different conformal field theory; thus the phase is unchanged but the particular fixed point is different.

- For $N_{f}=6$, the theory without the perturbation is in the free magnetic phase, but the perturbation drives the theory to an interacting fixed point and thus into the non-abelian coulomb phase.

- For $N_{f}=5$, the theory is confining; the perturbation obviously breaks some chiral symmetries, but even more are broken dynamically as a result of the perturbation.

\subsection{Concluding Comments}

Aside from the examples discussed here, many others are known. For most understood gauge theories, the phase diagrams - a free electric phase above, a conformal phase below, possibly a free magnetic phase and usually confining phases, with or without chiral symmetry breaking - are qualitatively similar, although the details differ in very interesting ways. Various new phenomena have been uncovered along the way. But most $\mathcal{N}=1$ supersymmetric field theories are not understood, and much work remains to be done.

The most remarkable aspect of these phase diagrams is that they show that the phase of a theory strongly depends on its gauge group $G$, its mass-

less matter representations $R$, and its interactions $\mathcal{L}_{\text {int }}$, renormalizable and 
non-renormalizable. The dependence on $R$ goes far beyond the mere contribution of the matter to the beta function; the matter fields are clearly more than spectators to the gauge dynamics. (A quenched approximation could not reproduce this phase structure.) The dependence on non-renormalizable interactions is familiar from technicolor theories: a higher-dimension operator, though irrelevant in the ultraviolet, may become relevant in the infrared and control the physics of the low-energy theory.

It is also worth noting that while calculational techniques exist for studying the infrared physics in most of these phases, the non-abelian coulomb phase requires an understanding of four-dimensional superconformal field theory. Techniques in this subject are still being explored ${ }^{25}$ and there is much left to be learned.

Given this complexity is present in SQCD, why should it not be found in non-supersymmetric gauge theories? The free electric phase, conformal or non-abelian coulomb phase, and confining/chiral-symmetry-breaking phase certainly arise. It would be remarkable indeed if the ubiquity of the conformal phase, the possibility of confinement without chiral symmetry breaking, and the existence of a free magnetic phase could be demonstrated. There might also be as yet unknown phases that do not occur in supersymmetric theories.

More specifically, we should seek to answer the following question: what is the phase of QCD as a function of $G, R$ and $\mathcal{L}_{\text {int }}$ ? Unfortunately the answer cannot be learned from the supersymmetric theories: the process of breaking supersymmetry leads to ambiguities in the duality transformations. We therefore need new tools, both analytical and numerical. This is clearly an area for lattice gauge theory, but it is not easy to study the renormalization group flow over large regions of energy using the lattice. Additional analytic work is needed to make this more tractable. I hope some readers will be motivated to consider this problem!

It should be stressed that this is not merely an academic question. It is possible that the correct theory of electroweak supersymmetry breaking (or of fermion masses, etc.) has not yet been written down. Perhaps a modified form of technicolor or something even more exotic will appear in the detectors of the Large Hadron Collider, in a form that we will be unable to understand unless the questions raised above are addressed in the coming years.

\section{Unification of Dualities in $\mathcal{N}=1$ Supersymmetry}

One of the most remarkable results of the recent developments is that many strong-coupling phenomena previously thought to be unrelated have turned out to be profoundly linked. This is best understood by looking at a variety of 
"duality transformations", non-local changes of variables which now seem to appear everywhere we look for them. Let us begin by listing a few:

Electric-Magnetic (EM): this is the usual duality transformation of the Maxwell equations without matter, which can be trivially extended to $\mathcal{N}=4$, 2,1 supersymmetric Maxwell theory. The electric and magnetic gauge groups are $U(1)_{e}$ and $U(1)_{m}$ (note these two symmetry groups are completely distinct transformations on the non-locally related electric and magnetic gauge potentials.) The electric and magnetic couplings are $e$ and $4 \pi / e$. (The last relation is modified for non-zero theta angle.)

Dual Meissner (DM): for abelian gauge theory, or for a non-abelian gauge theory which breaks down to an abelian subgroup. Such a theory often has magnetic monopoles, which are described as locally and minimally coupled to a magnetic photon. If the monopoles condense, breaking the magnetic gauge symmetry, the magnetic photon obtains a mass, screening magnetic flux and confining the electric flux of the original description of the theory.

Olive-Montonen ${ }^{26,27,28}(\mathrm{OM})$ : the EM case for $\mathcal{N}=4$ supersymmetry, extended to a non-abelian gauge group $G_{e}$. The magnetic variables also are an $\mathcal{N}=4$ supersymmetric gauge theory and have a gauge group $G_{m}$. The theory is conformal and has a quantum-mechanically dimensionless (non-running) coupling constant $g$. The coupling in the magnetic description of the theory is $4 \pi / g$. (The last relation is modified for non-zero theta angle.)

Generalized Dual Meissner ${ }^{29,21}$ (GDM): similar to the DM case, but where both the electric and magnetic gauge groups $G_{e}$ and $G_{m}$ are non-abelian. Condensing magnetically charged monopoles again break $G_{m}$, screen magnetic flux and confine electric flux.

Seiberg-Witten pure ${ }^{8}(\mathrm{SWp})$ : for pure $\mathcal{N}=2$ supersymmetric Yang-Mills theory. The electric theory has gauge group $G$ of rank $r$, whose maximal abelian subgroup is $\left[U(1)^{r}\right]_{e}$. EM duality applies to each $U(1)$; the magnetic theory has gauge group $\left[U(1)^{r}\right]_{m}$.

Seiberg-Witten finite ${ }^{30}$ (SWf): for a finite $\mathcal{N}=2$ supersymmetric gauge theory with matter. Very similar to OM above, but in general the relation between $G_{e}$ and $G_{m}$ differs from the OM case.

$\mathrm{QCD}$ and the Sigma Model (QCD $\sigma$ ): here a strongly-coupled, confining QCD or $\mathcal{N}=1$ SQCD theory is described in terms of gauge singlets, using a linear or non-linear sigma model. This is not always considered a "duality", but as we will see, it should be.

The main point of this section is to emphasize that all of these dualities are linked together ${ }^{10,16}$ by results in $\mathcal{N}=1$ supersymmetry. This can be easily seen using the duality of $\mathcal{N}=1 S O(N)$ gauge theories with $N_{f}$ fields 
in the vector representation; as mentioned earlier, such theories are dual to $S O\left(N_{f}-N+4\right)$ with $N_{f}$ vectors and $N_{f}\left(N_{f}+1\right) / 2$ gauge singlets. ${ }^{10,15}$

Consider first $S O(2)$ without matter $\left(N_{f}=0\right)$. The dual theory is again $S O(2)$ without matter - EM duality - which justifies referring to the dual theory as "magnetic".

Next, consider $S O(3)$ with one triplet; its magnetic dual is $S O(2)$ with fields of charge $1,0,-1$ coupled together. These theories are both $\mathcal{N}=2$ supersymmetric; the electric theory is the pure $\mathcal{N}=2 S U(2)$ theory studied by Seiberg and Witten, and the magnetic dual is the theory of the light monopole which serves as its low-energy description. ${ }^{8}$ Since a mass for the triplet leads to confinement of $S O(3)$ via abelian monopole condensation, ${ }^{8}$ this example gives both SWp and DM duality.

Finally, take $S O(3)$ with three triplets, whose dual is $S O(4) \approx S U(2) \times$ $S U(2)$, with three fields in the 4 representation and six singlets. If the triplets are massive, the quartets of the dual theory condense, $S O(4)$ is broken, and confinement of $S O(3)$ occurs ${ }^{10}$ - GDM duality. The low-energy description below the confinement scale is given by this broken $S O(4)$ theory ${ }^{10}$, a nonlinear sigma model - QCD $\sigma$ duality. And if instead the triplets are massless and are given the renormalizable interactions which make the theory $\mathcal{N}=4$ supersymmetric, the dual theory is consistent with OM duality: ${ }^{26,30}$ one of the $S U(2)$ subgroups of $S O(4)$ confines, leaving a single $S U(2)$ factor with three triplets coupled by the required $\mathcal{N}=4$ supersymmetric interactions. ${ }^{15,16}$

Thus, $\mathcal{N}=1$ duality links all of the four-dimensional dualities on the list together, showing they are manifestations of a single phenomenon. Figure 5 shows a cartoon of the connections between these theories. I have omitted, both from my list and from this figure, other types of field theory duality that were not known until recently. Also not shown are the relations between these dualities and those of the-theory-formerly-known-as-string theory, sometimes termed M theory. The situation brings to mind the old story of the blind men and the elephant, in which each man feels one part of the elephant - the ear, or the trunk, or the tail - and erroneously assigns it a special significance, not realizing that the apparently different parts are connected to a larger whole. Having discovered the existence of a larger whole, we find ourselves compelled to explain duality in a unified way. There have been some recent developments in this subject. ${ }^{31,32}$

I want to emphasize to those who are not familiar with this subject that this picture, while not proven, is by no means speculative. The circumstantial evidence in its favor - a vast number of consistency conditions - is completely overwhelming; one could easily give twenty lectures on this subject. A proof is still badly needed, however; the underlying meaning of duality remains 


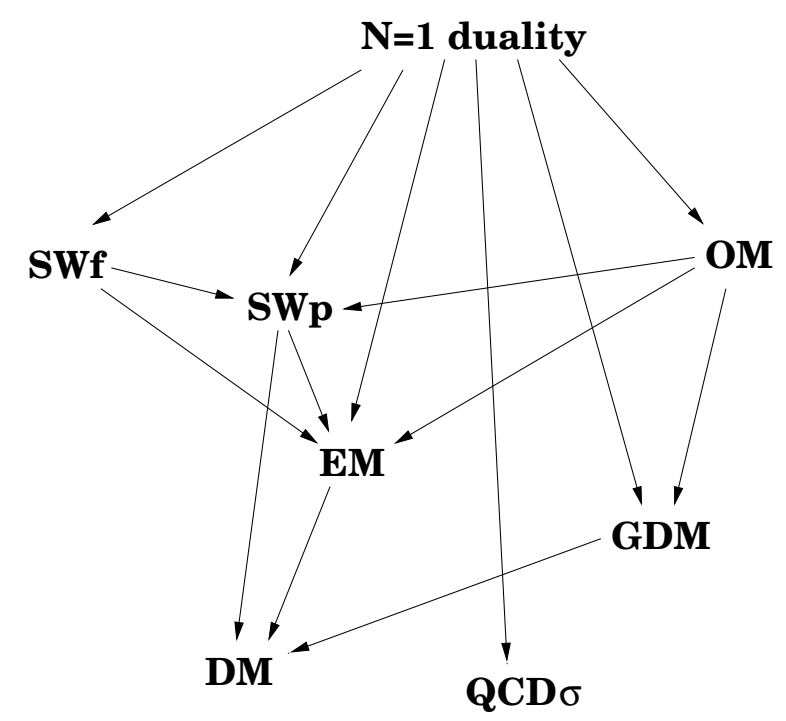

Figure 5: Interrelation between types of duality.

mysterious, and no field theoretic formulation is known which would make it self-evident (except in the EM case, of course.)

\section{Olive-Montonen Duality, Confinement, and the QCD String}

What drives confinement? In particular, why is $\mathcal{N}=1$ SYM confining? This question cannot be addressed directly in SYM, because this theory does not have a weakly-coupled dual description. However, there is a trick for studying this question - within limits, as discussed further below. The trick is the following. One can add additional massive matter to SYM without leaving its universality class (note supersymmetry and holomorphy ensure this.) ${ }^{7} \mathrm{In}$ particular I will study broken $\mathcal{N}=2 \mathrm{SYM}^{8,9}$ and broken $\mathcal{N}=4 \mathrm{SYM}^{33,29,22}$ gauge theories, which have the same massless fields as $\mathcal{N}=1 \mathrm{SYM}$. These theories have duality transformations which allow their dynamics to be studied using weakly-coupled magnetic descriptions. In these descriptions there are monopoles in the theory, which condense, thereby causing confinement. The confining flux tubes appear as string solitons. The picture which emerges appears to support the old lore that the Dual Meissner effect is responsible for confinement.

Before beginning the pedagogical presentation of these arguments, let me 


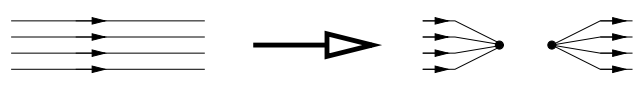

Figure 6: The annihilation of $N$ flux tubes using baryon vertices; this transition would be inhibited if a good dual description of YM theory involved $U(1)^{N-1}$.

summarize the results to be obtained below. The $\mathcal{N}=2 S U(N)$ SYM theory, with strong coupling scale $\Lambda$, has a dual description as an abelian $U(1)^{N-1}$ gauge theory; ${ }^{8,34}$ the monopoles of the $S U(N)$ description are electrically charged under the dual description. When masses $\mu \ll \Lambda$ are added to the extra matter, so that the only massless fields are those of $\mathcal{N}=1 \mathrm{SYM}$, the monopoles develop expectation values. The dual description of this condensation involves nothing more than $N-1$ copies of the Abelian Higgs model, and so gives $N-1$ solitonic Nielsen-Olesen strings, 8,9 each carrying an integer charge. Because of this monopole condensate, electrically charged sources of $S U(N)$ are confined, and the flux tubes which confine them are the solitonic strings of the dual description. However, the confining strings are problematic. ${ }^{9,35}$ Although they carry an exact $\mathbf{Z}_{N}$ symmetry, they also each carry an (approximate) integer charge, violated only at the scale $\Lambda$ which is large compared to the string tension. This extra symmetry leads the theory to exhibit not one but $N / 2$ metastable Regge trajectories - and thus the theory does not have the same properties as YM or $\mathcal{N}=1 \mathrm{SYM}$. As $\mu \rightarrow \Lambda$, the extra symmetry begins to disappear, but at the same time the magnetic description becomes strongly coupled, so no reliable dual description can be given in the regime where the theory behaves as $\mathcal{N}=1 \mathrm{SYM}$ is expected to do.

Note these properties are characteristic of abelian projection approaches to confinement. If we project $S U(N) \rightarrow U(1)^{N-1}$, dynamically or otherwise, then abelian monopole condensation leads to Nielsen-Olesen strings, each with its own approximately conserved integer charge. This unavoidable charge inhibits annihilation of $N$ identical strings (as in Fig. 6) which both leads to an overabundance of metastable hadrons ${ }^{a}$ and to difficulty in forming baryons. ${ }^{35}$ This is a serious problem for abelian projection approaches to YM and QCD.

The situation in broken $\mathcal{N}=4 \mathrm{SYM}^{22}$ is much more satisfactory. $\mathcal{N}=4$ $S U(N)$ SYM is a conformal field theory, with gauge coupling $g$. Its magnetic description, also an $\mathcal{N}=4$ conformal field theory, has gauge group $S U(N) / \mathbf{Z}_{N}$ and coupling $1 / g$; thus, if $g \gg 1$, the magnetic description is weakly coupled. Adding masses $\mu$ to all but the fields of $\mathcal{N}=1$ SYM causes the scalars of the magnetic description to condense, breaking the dual gauge

${ }^{a}$ Here and elsewhere, by "hadrons of Yang-Mills theory" I mean mesons and baryons built from quarks whose masses are large enough compared to $\Lambda$ that they do not greatly affect the infrared dynamics. 
group completely. ${ }^{33,29}$ This non-Abelian Higgs model has string solitons, but because the fundamental group of $S U(N) / \mathbf{Z}_{N}$ is $\mathbf{Z}_{N}$, these strings carry a $\mathbf{Z}_{N}$ charge, ${ }^{22}$ in contrast to the integer charges found in the case of broken $\mathcal{N}=2$ SYM. In short, the electric description of the theory involves confinement by electric flux tubes carrying $\mathbf{Z}_{N}$ electric flux, leading to a single Regge trajectory, in agreement with expectations for $\mathcal{N}=1 \mathrm{SYM}$. Notice that the associated dual description does not resemble abelian projection: it is essential for the $\mathbf{Z}_{N}$ charges of the strings that the dual theory is non-abelian. Since one cannot obtain the dual $S U(N) / \mathbf{Z}_{N}$ theory by a projection on the $S U(N)$ theory - the relation between the two is fundamentally quantum mechanical - it seems to me that abelian projection is conceptually disfavored.

Note however that one cannot carry this logic all the way to the $\mathcal{N}=1$ SYM theory itself. To do so requires taking $\mu \rightarrow \infty, g \rightarrow 0$, but in this limit the magnetic theory becomes strongly coupled and the semiclassical discussion of the previous paragraph becomes unreliable.

Thus, a strong word of caution is in order here. In particular, although both broken $\mathcal{N}=2$ and broken $\mathcal{N}=4$ are in the same universality class as $\mathcal{N}=1 \mathrm{SYM}$, they are not equivalent to it. While confinement and an energy gap are universal properties of all of these theories, the monopoles which lead to confinement are not universal. The properties of the monopoles depend on the matter that is added to SYM. Again, this poses problems for abelian projection approaches to confinement, or indeed for any semiclassical description of the phenomenon.

\subsection{Electric Sources and Fluxes}

I begin with a review of basic and well-known facts about gauge theories. Consider a pure gauge theory with gauge group $G$. Suppose we have a sourcean infinitely massive, static, electrically charged particle - in a representation $R$ of $G$. If we surround the source with a large sphere, what characterizes the flux passing through the sphere? If $G$ is $U(1)$, the flux measures the electric charge directly. However, in non-abelian gauge theories the gauge bosons carry charge. Since there may be a number (varying over time) of gauge bosons inside the sphere, the representation under which the charged objects in the sphere transform is not an invariant. But, by definition, the gauge bosons are neutral under the discrete group $C_{G}$, the center of $G$. It follows that the charge of $R$ under the center is a conserved quantity, and that the total flux exiting the sphere carries a conserved quantum number under $C_{G}$.

For example, in $S U(N)$ the center is a $\mathbf{Z}_{N}$ group with the lovely name of "N-ality". A tensor $T_{b_{1} b_{2} \cdots b_{q}}^{a_{1} a_{2} \cdots a_{p}}$ has N-ality $p-q \bmod N$; in particular, the 
$k$-index antisymmetric tensors carry $\mathrm{N}$-ality $k$.

Conclusion: Electric sources and fluxes in pure gauge theories carry a conserved $C_{G}$ quantum number. If the gauge group confines, then the confining electric flux tubes will also carry this quantum number.

If the theory also contains light matter charged under $C_{G}$ but neutral under a subgroup $C_{m}$ of $C_{G}$, then the above statements are still true with $C_{G}$ replaced with $C_{m}$. For example, if we take $S U(N)$ with massless fields in the $\mathbf{N}$ representation, then $C_{m}$ is just the identity, reflecting the fact that all sources can be screened and all flux tubes break. If we take $S O(10)$ with fields in the $\mathbf{1 0}$, then the center $\mathbf{Z}_{4}$ is replaced with spinor-number $\mathbf{Z}_{2}$. Sources in the 10 will be screened and have no flux tube between them, while sources in the $\mathbf{1 6}$ or $\overline{\mathbf{1 6}}$ will be confined by a single type of flux tube.

\subsection{Confinement and Flux Tubes}

Like pure YM, $\mathcal{N}=1 \mathrm{SYM}$ is confining; how does this discussion apply to it? Since the gauge bosons and gauginos are all in the adjoint representation, the electric flux tubes do carry charge in the center of the gauge group; for $S U(N)$ they carry quantum numbers $k$ in $\mathbf{Z}_{N}$. What properties of these strings could we hope to predict? The tension $T_{k}$ of an infinitely long confining string is a function of $k, N, \Lambda$, and we might hope to say something about it. First, note $T_{k}=T_{N-k}, T_{N}=0$ by $\mathbf{Z}_{N}$ symmetry. An electric source of charge $k$ (e.g. one in a $k$-index antisymmetric tensor representation) will be confined by a $k$-string (a string carrying $k$ units of flux) or by a set of strings whose charges add to $k \bmod N$. The ratio $T_{k} / T_{k^{\prime}}$ is a basic property of YM and SYM, as fundamental as the glueball spectrum and easier for theorists to estimate. I will discuss some predictions for these ratios later.

\subsection{Magnetic Sources and Fluxes}

Now let consider what happens to magnetic sources and fluxes in gauge theories. First, let's review some basic topology. The $p$-th homotopy group of a manifold $\mathcal{M}, \pi_{k}(\mathcal{M})$, is the group of maps from the $p$-sphere into $\mathcal{M}$, where we identify maps as equivalent if they are homotopic in $\mathcal{M}$. All we will need for present purposes are the following examples. Suppose a Lie group $G$ has rank $r$, so that its maximal abelian subgroup is $U(1)^{r}$; then

$$
\pi_{2}[G]=\mathbf{1} \Rightarrow \pi_{2}\left[G / U(1)^{r}\right]=\pi_{1}\left[U(1)^{r}\right]=\mathbf{Z} \times \mathbf{Z} \times \cdots \times \mathbf{Z} \equiv[\mathbf{Z}]^{r} .
$$

Similarly,

$$
\pi_{1}[G]=\mathbf{1} \Rightarrow \pi_{1}\left[G / C_{G}\right]=\pi_{0}\left[C_{G}\right]=C_{G}
$$


We will need to investigate both monopole solitons and string solitons below. The classic monopole soliton is that of 't Hooft and of Polyakov, which arises in $S U(2)$ broken to $U(1)$; in this case the important topological relation is $\pi_{2}[S U(2) / U(1)]=\pi_{1}[U(1)]=\mathbf{Z}$. This leads to a set of monopole solutions carrying integer charge. Note that the stability of, for example, the charge-two monopole solution against decay to charge-one monopoles is determined not by topology but by dynamics. The situation is similar for the Nielsen-Olesen magnetic flux tube of the abelian Higgs model; here the relevant topological relation is $\pi_{1}[U(1)]=\mathbf{Z}$. This again leads to solutions with an integer charge, whose stability against decay to minimally charged vortices is determined dynamically.

More generally, if we have a simply connected gauge group $G_{0}$ which breaks to a group $G$ at a scale $v$, there will be monopoles carrying a quantum number in $\pi_{2}\left[G_{0} / G\right]$, of mass [radius] proportional to $v[1 / v]$. Now imagine that we take $v \rightarrow \infty$. In this limit the gauge group $G_{0}$ disappears from the system. The monopoles become pointlike and infinitely massive; their only non-pointlike feature is their Dirac string, which carries a quantum number in $\pi_{1}[G]$. In short, the solitonic monopoles become fundamental Dirac monopoles in this limit. Note that since $\pi_{2}\left[G_{0} / G\right]=\pi_{1}[G]$, the charges carried by the solitonic monopoles and their Dirac monopole remnants are the same. Since the Dirac monopoles are heavy, we may use them as magnetic sources.

Let's further suppose that the gauge group $G$ is broken completely at some scale $v^{\prime}$. In this case no Dirac strings can exist in the low-energy theory, and so the monopoles allowed previously have seemingly vanished. However, solitonic magnetic flux tubes, carrying charges under $\pi_{1}[G]$, will be generated; they will have tension [radius] of order $v^{\prime 2}\left[1 / v^{\prime}\right]$. Their $\pi_{1}[G]$ quantum numbers are precisely the ones they need to confine the $\pi_{1}[G]$-charged Dirac monopole sources of the high-energy theory. Thus, when $G$ is completely broken, the Dirac monopoles disappear because they are confined by flux tubes.

Conclusion: Magnetic sources and fluxes in pure gauge theories carry a conserved $\pi_{1}[G]$ quantum number. If the gauge group is completely broken, then the confining magnetic flux tubes will also carry this quantum number.

\section{$5.4 \mathcal{N}=4$ Supersymmetric Gauge Theory}

The next ingredient in this stew is $\mathcal{N}=4$ supersymmetric gauge theory, consisting of one gauge field, four Majorana fermions, and six real scalars, all in the adjoint representation. It is useful to combine these using the language of $\mathcal{N}=1$ supersymmetry, in which case we have one vector multiplet (the gauge boson $A_{\mu}$ and one Majorana fermion $\lambda$ ) and three chiral multiplets (each with 
a Weyl fermion $\psi^{s}$ and a complex scalar $\Phi^{s}, s=1,2,3$.)

These fields have the usual gauged kinetic terms, along with additional interactions between the scalars and fermions. The scalars, in particular, have potential energy

$$
V\left(\Phi^{s}\right)=\sum_{a=1}^{\operatorname{dim} G}\left|D_{a}^{2}\right|+\sum_{s=1}^{3}\left|F_{s}\right|^{2}
$$

where

$$
D_{a}=\left(\sum_{s=1}^{3}\left[\Phi^{s \dagger}, \Phi^{s}\right]\right)_{a}
$$

(here $a$ is an index in the adjoint of $G$ ) and

$$
F_{s}=\epsilon_{s t u}\left[\Phi^{t}, \Phi^{u}\right]
$$

Supersymmetry requires that $\left\langle V\left(\Phi^{s}\right)\right\rangle=0$, and so all $D_{a}$ and $F_{s}$ must vanish separately. The solution to these requirements is that a single linear combination $\hat{\Phi}$ of the $\Phi^{s}$ may have non-vanishing expectation value, with the orthogonal linear combinations vanishing. By global rotations on the index $s$ we may set $\hat{\Phi}=\Phi^{3}$. By gauge rotations we may make $\Phi^{3}$ lie in the Cartan subalgebra of the group; we may represent it as a diagonal matrix

$$
\left\langle\Phi^{3}\right\rangle=\operatorname{diag}\left(v_{1}, v_{2}, \cdots\right)
$$

which (if the $v_{i}$ are all distinct) breaks $G$ to $U(1)^{r}$. Since $\pi_{2}\left[G / U(1)^{r}\right]=[\mathbf{Z}]^{r}$ [see Eq. (4)] the theory has monopoles carrying $r$ integer charges under $U(1)^{r}$. Quantum mechanically, the theory has both monopoles and dyons, carrying $r$ electric and $r$ magnetic charges $\left(n_{e}, n_{m}\right)$.

The space of vacua written in Eq. (9) is not altered by quantum mechanics. In the generic $U(1)^{r}$ vacuum, each $U(1)$ has EM duality. In a vacuum where some of the $v_{i}$ are equal, the gauge group is broken to a non-abelian subgroup $\hat{G}$ times a product of $U(1)$ factors; the low-energy limit of the non-abelian part is an interacting conformal field theory. The $U(1)$ factors have EM duality, while the $\hat{G}$ factor has its non-abelian generalization, OM duality. ${ }^{26,27,28,30}$

\subsection{Olive-Montonen Duality}

The $\mathcal{N}=4$ theory has a set of alternate descriptions, generated by changes of variables (whose explicit form remains a mystery) of the form

$$
\mathbf{T}: \tau \rightarrow \tau+1(\theta \rightarrow \theta+2 \pi) ; n_{e} \rightarrow n_{e}+n_{m}, n_{m} \rightarrow n_{m} ; G \rightarrow G ;
$$


and

$$
\mathbf{S}: \tau \rightarrow-\frac{1}{\tau}\left(g \rightarrow \frac{4 \pi}{g} \text { if } \theta=0\right) ; n_{e} \leftrightarrow n_{m} ; G \rightarrow \tilde{G} .
$$

Together $\mathbf{S}$ and $\mathbf{T}$ generate the group $S L(2, \mathbf{Z})$, which takes $\tau \rightarrow(a \tau+b) /(c \tau+$ $d)$ for integers $a, b, c, d$ satisfying $a d-b c=1$. Note that $\mathbf{T}$ is nothing but a rotation of the theta angle by $2 \pi$; it does not change the gauge group or the definition of electrically charged particles, shifting only the electric charges of magnetically charged particles ${ }^{36}$ By contrast, $\mathbf{S}$ exchanges electric and magnetic charge, weak and strong coupling (if $\theta=0$ ) ${ }^{26}$ and changes the gauge group ${ }^{27,28}$ from $G$ to its dual group $\tilde{G}$, as defined below.

The group $G$ has a root lattice $\Lambda_{G}$ which lies in an $r=\operatorname{rank}(G)$ dimensional vector space. This lattice has a corresponding dual lattice $\left(\Lambda_{G}\right)^{*}$. It is a theorem that there exists a Lie group whose root lattice $\Lambda_{\tilde{G}}$ equals $\left(\Lambda_{G}\right)^{*} \cdot{ }^{27}$ Here are some examples:

$$
\begin{array}{cl}
S U(N) \leftrightarrow S U(N) / \mathbf{Z}_{N} ; & S O(2 N+1) \leftrightarrow U S p(2 N) ; \\
S O(2 N) \leftrightarrow S O(2 N) ; & \operatorname{Spin}(2 N) \leftrightarrow S O(2 N) / \mathbf{Z}_{2} .
\end{array}
$$

Notice that this set of relationships depends on the global structure of the group, not just its Lie algebra; $S O(3)$ (which does not have spin- $1 / 2$ representations) is dual to $U S p(2) \approx S U(2)$ (which does have spin- $1 / 2$ representations.) These details are essential in that they affect the topology of the group, on which OM duality depends.

In particular, there are two topological relations which are of great importance to OM duality. The first is relevant in the generic vacuum, in which $G$ is broken to $U(1)^{r}$. The electric charges under $U(1)^{r}$ of the massive electrically charged particles $\left(\operatorname{spin} 0, \frac{1}{2}, 1\right)$ lie on the lattice $\Lambda_{G}$. The massive magnetic monopoles (also of spin $0, \frac{1}{2}, 1$ ) have magnetic charges under $U(1)^{r}$ which lie on the dual lattice $\left(\Lambda_{G}\right)^{*} 2^{27,28}$ Clearly, for the $\mathbf{S}$ transformation, which exchanges the electrically and magnetically charged fields and the groups $G$ and $\tilde{G}$, to be consistent, it is essential that $\Lambda_{\tilde{G}}=\left(\Lambda_{G}\right)^{*}$ — which, fortunately, is true.

The second topological relation is the one we will use below. We have seen that the allowed electric and magnetic sources for a gauge theory with adjoint matter (such as $\mathcal{N}=4$ ) are characterized by quantum numbers in $C_{G}$ and $\pi_{1}(G)$ respectively. Consistency of the $\mathbf{S}$ transformation would not be possible were these two groups not exchanged under its action. Fortunately, it is a theorem of group theory that ${ }^{27}$

$$
\pi_{1}(G)=C_{\tilde{G}} ; \pi_{1}(\tilde{G})=C_{G} .
$$

For example, $\pi_{1}[S U(N)]=C_{S U(N) / \mathbf{Z}_{N}}=\mathbf{1}$ while $C_{S U(N)}=\pi_{1}\left[S U(N) / \mathbf{Z}_{N}\right]=$ $\mathbf{Z}_{N}$. 
Conclusion: As a consequence of Eq. (13) and the results of sections 5.1 and 5.2 , the allowed magnetic sources of $G$ are the same as the allowed electric sources for $\tilde{G}$, and vice versa.

\subsection{Breaking $\mathcal{N}=4$ to $\mathcal{N}=1$}

Now, we want to break $\mathcal{N}=4$ supersymmetry to $\mathcal{N}=1$. Pure $\mathcal{N}=1 \mathrm{SYM}$, like pure non-supersymmetric YM, is a confining theory, and should contain confining flux tubes. The addition of massive matter in the adjoint representation does not change this; heavy particles would only obstruct confinement by breaking flux tubes, which adjoint matter cannot do. We therefore expect that broken $\mathcal{N}=4$ gauge theory, which is $\mathcal{N}=1 \mathrm{SYM}$ plus three massive chiral fields in the adjoint representation, should be in the same universality class as pure SYM: both should confine, and both should have flux tubes carrying a $C_{G}$ quantum number, as discussed in section 5.1.

We may break the $\mathcal{N}=4$ symmetry by adding masses $m_{s}$ for the fields $\Phi^{s}$; the $F_{s}$ functions of (8) become

$$
F_{s}=\epsilon_{s t u}\left[\Phi^{t}, \Phi^{u}\right]+m_{s} \Phi^{s}
$$

so that $F_{s}=0$ implies $\epsilon_{s t u}\left[\Phi^{t}, \Phi^{u}\right]=-m_{s} \Phi^{s}$. Up to normalization these are the commutation relations for an $S U(2)$ algebra, ${ }^{33}$ which I will call $S U(2)_{\text {aux }}$. If we take $m_{1}=m_{2}=m$ and $m_{3}=\mu$ we obtain

$$
\Phi^{1}=-i \sqrt{\mu m} J_{x} ; \Phi^{2}=-i \sqrt{\mu m} J_{y} ; \Phi^{3}=-i m J_{z},
$$

where $J_{x}, J_{y}, J_{z}$ are matrices satisfying $\left[J_{x}, J_{y}\right]=i J_{z}$, etc. Each possible choice for the $J$ 's gives a separate, isolated vacuum. ${ }^{33}$

How does this work, explicitly, in $S U(N)$ ? We can write the $\Phi^{s}$ as $N \times N$ traceless matrices, so the $J_{i}$ should be an $N$-dimensional (generally reducible and possibly trivial) representation of $S U(2)_{\text {aux }} \cdot{ }^{33,29}$ The trivial choice corresponds to $J_{i}=0$; clearly if $\Phi^{s}=0$ the $S U(2)_{\text {aux }}$ commutation relations are satisfied. We will call the corresponding vacuum the "unbroken" vacuum, since the $S U(N)$ gauge group is preserved. Another natural choice is to take the $J_{i}$ in the irreducible spin- $\frac{N-1}{2}$ representation of $S U(2)_{\text {aux }}$. In this case $S U(N)$ is completely broken; we will call this the "Higgs vacuum". We may also choose the $J_{i}$ in a reducible representation such as

$$
J_{i}=\left[\begin{array}{ccc}
\sigma_{i} & \mid & 0 \\
-- & -\mid- & -- \\
0 & \mid & 0
\end{array}\right] \text {; }
$$


here the $\sigma_{i}$ are the Pauli matrices. In this case $S U(N)$ is partly broken. There are many vacua like this last one, but they will play no role in the physics below; we will only need the unbroken vacuum and the Higgs vacuum.

Conclusion: The classical analysis of this $\mathcal{N}=1$ supersymmetric $S U(N)$ gauge theory with massive adjoint fields shows that it has isolated supersymmetric vacua scattered about, with the unbroken $(\mathrm{U})$ vacuum at the origin of field space and the Higgs vacuum $(\mathrm{H})$ at large $\Phi^{s}$ expectation values [of order $m, \sqrt{m \mu}$, see Eq. (15).] $]^{33,29}$

\subsection{OM Duality and the Yang-Mills String}

The above picture is modified by quantum mechanics. In each vacuum, strong dynamics causes confinement to occur in the unbroken non-abelian subgroup, modifying the low-energy dynamics and generally increasing the number of discrete vacua. In the $\mathrm{H}$ vacuum, the gauge group is completely broken and no non-trivial low-energy dynamics takes place; it remains a single vacuum. The $\mathrm{U}$ vacuum, by contrast, splits into $N$ vacua - the well-known $N$ vacua of $\mathrm{SYM}^{37}$ — which I will call $\mathrm{D}_{0}, \mathrm{D}_{1}, \cdots, \mathrm{D}_{N-1}$. In the $\mathrm{D}_{k}$ vacuum, confinement occurs by condensation of dyons of magnetic charge 1 and electric charge $k^{8,29,9,34}$ Since these vacua are related ${ }^{37}$ by rotations of $\theta$ by multiples of $2 \pi$, I will focus on just one of them. It is convenient to study the $\mathrm{D}_{0}$ vacuum (which $\mathrm{I}$ now rename the $M$ vacuum) in which electric charge is confined by magnetic monopole condensation.

Now, what is the action of OM duality on this arrangement? The vacua are physical states, and cannot be altered by a mere change of variables; however, the description of each vacuum will change. Specifically, when $\theta=0$, the $\mathbf{S}$ transformation, which inverts the coupling constant and exchanges electric and magnetic charge, exchanges the $\mathrm{H}$ vacuum of $S U(N)$ for the M vacuum of $S U(N) / \mathbf{Z}_{N}$ and vice versa. ${ }^{29}$ To say it another way, the confining M vacuum of $S U(N)$ can be equally described as the $\mathrm{H}$ vacuum of $S U(N) / \mathbf{Z}_{N}$, in which the monopoles of the $S U(N)$ description break the dual $S U(N) / \mathbf{Z}_{N}$ gauge group. This is the Generalized Dual Meissner effect, in which both the electric and magnetic gauge groups are non-abelian.

Conclusion: $\mathrm{OM}$ duality exchanges the $\mathrm{H}$ and $\mathrm{M}$ vacua of $S U(N)$ broken $\mathcal{N}=4$ with the $\mathrm{M}$ and $\mathrm{H}$ vacua of $S U(N) / \mathbf{Z}_{N}$ broken $\mathcal{N}=4$. Confinement in the M vacuum of $S U(N)$ is described as the breaking of the $S U(N) / \mathbf{Z}_{N}$ gauge group in $S U(N) / \mathbf{Z}_{N}{ }^{29}$

The existence of the Yang-Mills string now follows directly from topology. As we discussed in section 5.2, the complete breaking of a group $G$ leads to solitonic strings carrying magnetic flux with a quantum number in $\pi_{1}(G)$. In 
this case, the breaking of $S U(N) / \mathbf{Z}_{N}$ in its $\mathrm{H}$ vacuum (the confining $\mathrm{M}$ vacuum of $S U(N))$ gives rise to strings with a $\mathbf{Z}_{N}$ quantum number. But magnetic flux tubes of $S U(N) / \mathbf{Z}_{N}$ are, by OM duality, electric flux tubes of $S U(N)$ - and so the confining strings of the $S U(N)$ theory's M vacuum, the confining theory which is in the same universality class as $S U(N)$ SYM, carry a $\mathbf{Z}_{N}$ quantum number. This is in accord with the considerations of section 5.1. The relation Eq. (13) is responsible for this agreement of the $\mathbf{Z}_{N}$ charges, and presumably assures a similar agreement for all groups.

Conclusion: OM duality gives a new picture for confinement in $S U(N)$ SYM: it occurs via non-abelian dual monopole condensation, and leads to confining strings with a $\mathbf{Z}_{N}$ quantum number.

A cautionary remark is in order. The description of confinement via dual monopole condensation is not fully reliable, as it is only appropriate if the $S U(N) / \mathbf{Z}_{N}$ theory is weakly coupled. In fact, we want the $S U(N)$ theory to be weakly coupled in the ultraviolet, in analogy with QCD. The $\mathbf{S}$ transformation implies that the $S U(N) / \mathbf{Z}_{N}$ description should be strongly coupled in the ultraviolet. However, the existence of a soliton carrying a stable topological charge is more reliable, especially since there are no other objects carrying that charge into which these string solitons can decay. Having constructed the solitonic strings semiclassically in some regime, we expect that they survive into other regimes in which semiclassical analysis would fail. (A gap in the argument: could the strings grow large and have zero string tension in the SYM limit?) In short, while the condensing monopole description appropriate to broken $\mathcal{N}=4 \mathrm{SYM}$ may not be valid for pure $\mathcal{N}=1 \mathrm{SYM}$, it does demonstrate the presence of confining strings in the latter. Whether anything quantitative can be said about these strings is another matter, to be addressed below.

Should we expect this picture to survive to the non-supersymmetric case? Take the theory with $\mathcal{N}=4$ supersymmetry broken to $\mathcal{N}=1$, and futher break $\mathcal{N}=1$ supersymmetry by adding an $S U(N)$ gaugino mass $m_{\lambda} \ll m, \mu$. We cannot be sure of the effect on the dual $S U(N) / \mathbf{Z}_{N}$ theory; duality does not tell us enough. However, we know that the theory has a gap, so this supersymmetry-breaking can only change some properties of the massive fields, without altering the fact that $S U(N) / \mathbf{Z}_{N}$ is completely broken. The strings, whose existence depends only on this breaking, thus survive for small $m_{\lambda}$. To reach pure YM requires taking $m, \mu, m_{\lambda}$ all to infinity. It seems probable, given what we know of YM physics, that the strings undergo no transition as these masses are varied. In particular, there is unlikely to be any phase transition for the strings between pure SYM and pure YM; this conjecture can and should be tested on the lattice.

Conclusion: If the strings of SYM and of YM are continously related, 
without a transition as a function of the gaugino mass, then the arguments given above for SYM extend to YM, establishing a direct link between OM duality of $\mathcal{N}=4$ gauge theory and the confining $\mathbf{Z}_{N}$-strings of pure YM.

\subsection{Confinement According to Seiberg and Witten}

How does this picture of confinement differ from that of Seiberg and Witten? Where and why might it be preferable?

Seiberg and Witten studied pure $\mathcal{N}=2$ supersymmetric $S U(2)$ gauge theory. ${ }^{8}$ They showed that the infrared quantum mechanical theory could be understood as a $U(1)$ theory coupled to a magnetic monopole, and that, when $\mathcal{N}=2$ supersymmetry is broken to $\mathcal{N}=1$, the monopole condenses, confining the $S U(2)$ degrees of freedom. The picture generalizes ${ }^{34}$ to $S U(N)$, where the infrared physics involves $U(1)^{N-1}$ coupled to $N-1$ monopoles, whose condensation drives confinement. This was studied in detail by Douglas and Shenker.

This physics is contained in a particular regime of the broken $\mathcal{N}=4$ supersymmetry gauge theory discussed above. If we take $\mu=0$, then the theory is $\mathcal{N}=2$ supersymmetric, and, as seen from Eq. (15), the $\mathrm{H}$ vacuum of $S U(N) / \mathbf{Z}_{N}$ has its gauge group broken only to $U(1)^{N-1}$. Now take $m$ exponentially large and the coupling at the scale $m$ small, so that the strong coupling scale $\Lambda$ of the low-energy theory is finite, and let $\mu$ be non-zero but small compared to $\Lambda$. The low-energy confining vacua of the $S U(N)$ theory will then be the vacua studied by Douglas and Shenker. The magnetic description of the theory (using OM duality) will have the gauge group $S U(N) / \mathbf{Z}_{N}$ broken at a high scale to $U(1)^{N-1}$, which in turn is broken completely at a low scale; see Eq. (15). The second step in this breaking leads to confinement of $S U(N)$ fields. If we take $m$ to infinity, then the $S U(N) / \mathbf{Z}_{N}$ gauge group disappears from the theory. The magnetic theory is merely $U(1)^{N-1}$ broken to nothing, as in Douglas and Shenker. ${ }^{34,9}$ (Note that I am cheating a bit here, as the abelian theory requires a cutoff; I'll fix this below.)

Since the magnetic theory is $U(1)^{N-1}$, dual to the maximal abelian subgroup of $S U(N)$, the pure $\mathcal{N}=2$ theory exhibits a dynamical form of abelian projection. ${ }^{9}$ The monopoles, whose condensation drives confinement when $\mathcal{N}=$ 2 supersymmetry is weakly broken, are purely abelian. Given the long-standing arguments using the monopoles of abelian projection as a mechanism for explaining confinement, why should this disturb us?

The problem lies with the quantum numbers of the strings. The $U(1)^{N-1}$ magnetic theory consists of $N-1$ copies of the abelian Higgs model, each of which has a Nielsen-Olesen solitonic flux tube. ${ }^{9}$ These strings carry quantum 


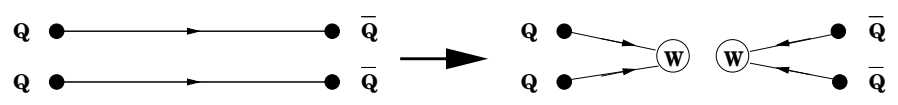

Figure 7: A pair of parallel $S U(2)$ strings can break via $W$ boson production.

numbers in the group $\pi_{1}\left[U(1)^{N-1}\right]=[\mathbf{Z}]^{N-1}$, not in $\mathbf{Z}_{N}$ ! That is, each of the $N-1$ Nielsen-Olesen strings carries its own conserved integer charge. These strings cannot lead to a good model for the dynamics of $S U(N)$ SYM or YM, which on general grounds must have $\mathbf{Z}_{N}$-carrying strings.

Is this problem serious? At first glance, the little cheat that I made just a moment ago rescues the abelian projection. The magnetic $U(1)^{N-1}$ theory has a cutoff at the scale $\Lambda$, where its coupling becomes large. At that scale, there are massive electrically-charged gauge bosons of $S U(N)$. Pair production of these particles cause certain configurations of parallel strings, which would naively be stable according to the reasoning of the previous paragraph, to break. The charges of these gauge particles are precisely such that they reduce the conserved symmetry from $[\mathbf{Z}]^{N-1}$ to $\mathbf{Z}_{N}$. For example, consider the case of $S U(2)$, as shown in figure 7 . An isospin- $\frac{1}{2}$ quark and a corresponding antiquark will be joined by a Nielsen-Olesen string. This string is stable. However, two such quark-antiquark pairs, with parallel strings, are unstable to reconfiguring their strings via $W$ boson production. This reflects the claim above that $W$ production reduces the symmetry under which the $S U(2)$ strings transform from $\mathbf{Z}$ to $\mathbf{Z}_{2}$.

Topologically speaking, all seems well - the charges seem to be the expected ones - but the dynamics of the theory still poses a serious problem. Although $[\mathbf{Z}]^{N-1}$ is not exactly conserved, it is approximately conserved. To see this in the $S U(2)$ example, note that the $W$ pair-production requires an energy of order $\Lambda$. The string tension (its energy per unit length) is $T=R_{\text {conf }}^{-2} \approx \mu \Lambda$ in this theory; here $R_{\text {conf }}$ is the confinement length. In order for the string to have enough energy to break, it should have length $L$ such that $T L \sim \Lambda$. This implies

$$
L \geq \frac{1}{\mu} \gg \frac{1}{\sqrt{\mu \Lambda}}=R_{\text {conf }},
$$

so only enormously long strings can break. Furthermore, since the strings' energy density is very low, it takes a large fluctuation to generate $W$ boson pairs. This in turn means that the rate for the transition in figure 7 is very slow. In short, the string pair shown in the figure is metastable for $\mu \ll \Lambda$. The $\mathbf{Z}$ symmetry is still approximately conserved by the dynamics. Similar 
arguments apply for $S U(N)$.

Conclusion: The strings of weakly broken $\mathcal{N}=2 S U(N)$ SYM carry an approximately conserved $[\mathbf{Z}]^{N-1}$ symmetry, which contains the expected $\mathbf{Z}_{N}$ as an exactly conserved subgroup. This leads to metastable string configurations not expected in $\mathcal{N}=1 \mathrm{SYM}$ and in YM.

The physical consequences of this approximately conserved symmetry are potentially dramatic. In SYM, a pair of parallel strings which carry fluxes of charge $k$ and $p$ under $\mathbf{Z}_{N}$ should undergo a rapid transition to a string carrying charge $k+p \bmod N$. (In QCD, this implies that the string between a quark and an antiquark is the same as the string between a quark and a diquark.) But this transition is inhibited in the broken $\mathcal{N}=2$ gauge theories for small $\mu$. This implies that numerous, distinct, metastable configurations of strings may connect a quark in the $\mathbf{N}$ representation to a corresponding antiquark. For example, for $k=1, \cdots N / 2$, a pair of parallel strings, with charges $k$ and $N-k+1$ respectively, carry total charge 1 ; they therefore may, as a pair, join a quark and antiquark. (Since a string with charge $N$ is no string at all, the $k=1$ case is the expected one.) These $N / 2$ metastable configurations have different energies per unit length, and in principle can give physically distinct quark-antiquark meson Regge trajectories.

Indeed, in $\mathcal{N}=2 \mathrm{SYM}$, the dynamics of the theory breaks the Weyl group, so the $N$ colors of quark are inequivalent. As shown by Douglas and Shenker, each color of quark prefers a different choice of string pairs. ${ }^{9}$ This leads to their most surprising conclusion.

Conclusion: In weakly broken $\mathcal{N}=2$ supersymmetric $S U(N)$ gauge theory, the quark-antiquark mesons exhibit $N / 2$ Regge trajectories, ${ }^{9}$ instead of one as expected in $\mathcal{N}=1 \mathrm{SYM}$ and in YM.

What happens to these extra trajectories as $\mu \rightarrow \infty$ and the theory approaches pure $\mathcal{N}=1$ SYM? As can be seen from Eq. (17), the obstructions to $W$ pair production go away as $\mu \rightarrow \Lambda$. (Note the formulas which lead to (17) receive corrections at order $\mu / \Lambda$.) The extra Regge trajectories become highly unstable and disappear from the spectrum. ${ }^{9}$ There is no sign of conflict with the usual SYM expectations of a single Regge trajectory and of strings with a $\mathbf{Z}_{N}$ symmetry. However, the $U(1)^{N-1}$ magnetic theory which we used to describe the weakly broken $\mathcal{N}=2$ theory becomes strongly coupled in this limit, and so one cannot study this picture quantitatively.

In summary, although broken $\mathcal{N}=2$ supersymmetric gauge theory can be used to show that $\mathcal{N}=1 \mathrm{SYM}$ is a confining theory, ${ }^{8,34,9}$ it is not a good model for the glueballs and heavy-quark hadrons of $\mathcal{N}=1 \mathrm{SYM}$. This is a direct consequence of the dynamical abelian projection, which leads to an abelian dual description. The condensation of its abelian monopoles leads to confinement by 
Nielsen-Olesen strings, which carry (approximately) conserved integer charges that (S)YM strings do not possess. These charges alter the dynamics of bound states, leading to a spectrum and to hadron-hadron interactions very different from those expected in SQCD and found in QCD. By contrast, these problems are avoided in the broken $\mathcal{N}=4$ description of confinement given in sections 5.5-5.6.

Moral: The use of abelian projection, and the construction of a dual abelian gauge theory, has inherent difficulties in explaining the dynamics of YM strings. One must therefore use abelian projection with caution. It may provide good answers for a limited set of questions, but for other questions it may fail badly.

\subsection{String tensions in $S U(N)$}

The discussion to this point has been entirely qualitative. Are any quantitative predictions possible?

A very useful theoretical quantity to study is the ratio of tensions of strings carrying different charge under $\mathbf{Z}_{N}$. A confining string of $S U(N)$ SYM or YM with quantum number $k$ under $\mathbf{Z}_{N}$ has a tension $T_{k}$ which depends on $k, N$ and the strong coupling scale $\Lambda$. On dimensional grounds $T_{k}=\Lambda^{2} f(k, N)$. While no analytic technique is likely to allow computation of $T_{1}$, it is possible that $T_{k} / T_{1}$ can be predicted with some degree of accuracy. Note that charge conjugation implies $T_{k}=T_{N-k}$, so $S U(2)$ and $S U(3)$ have only one string tension. We must consider $S U(4)$ and higher for this to be non-trivial.

While the ratio of tensions cannot be computed in continuum SYM or YM, it has been calculated in theories which are believed to be in the same universality class. These theories often have multiple mass scales $\mu_{i}$ and thus in principle it may be that $T_{k}=\Lambda^{2} h\left(\mu_{i} / \Lambda, k, N\right)$. However, in all cases studied so far, it has been found that $T_{k}=g\left(\mu_{i}, \Lambda\right) f(k, N)$, where $f$ is dimensionless, and $g$ is a dimensionful function which is independent of $k$. Note $g$ cancels out in ratios of tensions. Thus our attention may focus on the dimensionless function $f(k, N)$ as a quantity which may be compared from theory to theory.

Some previous calculations include the well-known strong-coupling expansion of YM, which to leading order gives

$$
f_{s c}(k, N) \propto k(N-k),
$$

and the results of Douglas and Shenker for weakly broken $\mathcal{N}=2$ gauge theory ${ }^{9}$

$$
f_{D S}(k, N) \propto \sin \frac{\pi k}{N} .
$$

The considerations of sections 5.5-5.6 suggest another calculation: if the string

solitons in broken $\mathcal{N}=4 S U(N)$ gauge theory were computed, the ratios of 
their tensions would be of considerable interest. At the time of writing these soliton solutions have not appeared in the literature.

There is one other technique by which string soliton tensions may be computed, using $\mathrm{M}$ theory! $\mathrm{M}$ theory is eleven-dimensional supergravity coupled to two-dimensional membranes. The theory also has five-dimensional solitons, called "five-branes", whose worldvolume is six-dimensional. The theory on the worldvolume of the five-brane is poorly understood but is known to be a $5+1$ dimensional conformal field theory. One can construct five-branes with rather strange shapes whose worldvolume theory contains, at low-energy, a sector with the same massless fields and interactions as $\mathcal{N}=2 S U(N) \mathrm{SYM}$, or broken $\mathcal{N}=2 S U(N) \mathrm{SYM}$, or pure $\mathcal{N}=1 \mathrm{SYM}$, or even (in principle) pure non-supersymmetric YM. (These theories also contain an infinite tower of massive particles, all neutral under $\mathbf{Z}_{N}$; thus they are potentially in the same universality class as, but should not be confused with, the gauge theories we are interested in. ${ }^{38}$ ) Witten showed membranes can bind to these five-branes, making objects that carry a $\mathbf{Z}_{N}$ charge and look in $3+1$ dimensions like strings. ${ }^{38}$ It was then shown ${ }^{35}$ that these strings indeed confine quarks and reproduce the results of Douglas and Shenker in the appropriate limit. However, the string tension ratios can be computed (naively) even when the $\mathcal{N}=2$ breaking parameter $\mu$ is large. One finds that the tensions are given by ${ }^{35}$

$$
T_{k}=g\left(\mu, \Lambda, R_{0}\right) f_{D S}(k, N)
$$

where $g$ is a complicated dimensionful function which cancels out of tension ratios, and $f_{D S}$ is as in Eq. (19). Thus, $M$ theory suggests that the tensions ratios satisfy (19) for large as well as small breaking of $\mathcal{N}=2$ supersymmetry. One must be careful with this result, however ${ }^{35,39}$ No non-renormalization theorem protects the result $(20)$ when the $\mathcal{N}=2$-breaking parameters are large. The overall coefficient function $g$ is certainly renormalized. The question is whether $f$ is strongly renormalized or not. Only a lattice computation will resolve this issue.

In summary, we so far have results on confining strings from the strong coupling expansion on the lattice (which only exists for non-supersymmetric theories), from broken $\mathcal{N}=2$ supersymmetric gauge theory in the continuum, and from the M theory versions of YM and SYM. There are a couple of interesting observations worth making.

First, the functions $f_{s c}$ and $f_{D S}$ have different large $N$ behavior. They agree at leading order, but while the first correction is at order $1 / N$ for $f_{s c}$ (as we would expect for an $S U(N)$ theory), the first correction for $f_{D S}$ is order $1 / N^{2}$ ! The fact that the $1 / N$ correction in $f_{D S}$ vanishes is surprising, and the physics that lies behind this feature has not been explained. 
Second, and more important, each calculable limit gives $T_{k}$ as a concavedown function of $k$, with $T_{k}<k T_{1}$ for all $k$; thus in each case a string with charge $k \leq N / 2$ is stable against decay to one or more strings of smaller charge. In short, flux tubes of small charge attract one another and form tubes of larger charge. In all of these limits, then, the gauge theory is a type I dual superconductor. I believe this result is robust and will be confirmed numerically in both SYM and YM. Specifically, I personally expect that $2 T_{1}>$ $T_{2}>T_{1}$ for $S U(N), N \geq 4$. The reasoning for this is the following. For $N=2$, we have $T_{2}=0$, while for $N=3$ we have $T_{2}=T_{1}, T_{3}=0$ on general grounds. For large $N$, we would expect $T_{k}=k T_{1} \pm \mathcal{O}(1 / N)$ since a $k$-string is a bound (or unbound) state of $k$ 1-strings, but string-string interactions are of order $1 / N$. Any reasonable interpolating formula will satisfy $k T_{1}>T_{k}>T_{1}$ for $N>4,1<k<N-1$. Inspired by this argument, Ohta and Wingate ${ }^{40}$ have compared $T_{1}$ and $T_{2}$ in $S U(4)$ by computing the potential energy between sources in the 4 and $\overline{4}$ representation $\left(V_{4 \overline{4}}(r) \sim T_{1} r\right)$ and the energy between sources in the 6 representation $\left(V_{66}(r) \sim T_{2} r\right.$. $)$ Their results suggest indeed that $2 T_{1}>T_{2}>T_{1}$.

\subsection{Discussion}

What has been obtained here? Two qualitatively different descriptions of confinement have been found, and neither can be continued directly to the theory of interest. One looks similar to abelian projection, while the other absolutely does not. What are we to make of this situation, and how are we to reconcile apparent contradictions?

I believe the correct way to view this situation ${ }^{b}$ is the following. Consider the space of theories in the same universality class as SYM, Fig. 8. Although all of these have a gap, confinement, and chiral symmetry breaking, only theories near a phase boundary, at the edge of the space, may be expected to have a weakly-coupled Landau-Ginsburg-type description. These dual descriptions may be used to establish the universal properties of $\mathcal{N}=1 \mathrm{SYM}$. Theories far from the boundary, such as $\mathcal{N}=1$ SYM itself, may simply not have any such description, and so there may not be any weakly-coupled effective theory for describing the string charges, hadron spectrum, and confinement mechanism of SYM. The same logic may apply to non-supersymmetric YM. ${ }^{c}$

${ }^{b}$ I thank N. Seiberg for discussions on this point.

${ }^{c}$ Note that it is very helpful, in the supersymmetric case, that the vanishing energy of the vacuum and holomorphy in the parameters of the Lagrangian ensure that there are no phase boundaries other than the one I have drawn in Fig. 8.7 Whether this is true in the nonsupersymmetric case must be tested numerically. 


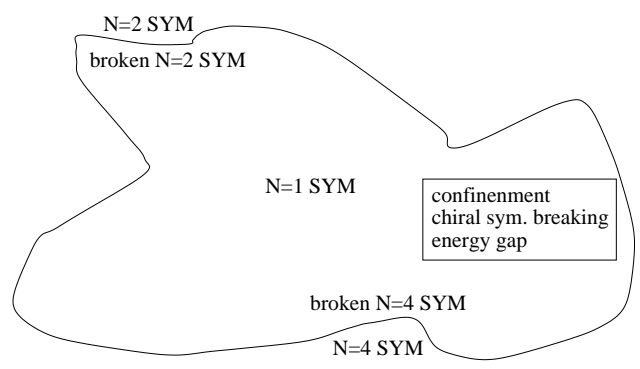

Figure 8: The universality class containing $\mathcal{N}=1 \mathrm{SYM}$, with $\mathcal{N}=2, \mathcal{N}=4 \mathrm{SYM}$ lying just outside.

In the end, then, our conclusions are very weak - we can show SYM is confining, but we cannot really say much about the mechanism which confines it. Any monopole description of confinement in SYM or YM is likely to be strongly coupled. Is such a description useful? or unambiguous? It seems unlikely to be predictive, in any case. It may be disappointing, but it appears likely there is no simple magnetic description of confinement in nature.

\section{Connecting to Non-Supersymmetric Theories}

Most physicists outside of the field of supersymmetry are inclined to think of supersymmetric theories, especially those with extended supersymmetry, as esoteric curiosities with no real importance for physics. I now intend to convince you that this is far from the truth. In fact, I will now argue that there is a direct link between the spectacular properties of $\mathcal{N}=4 \mathrm{SYM}$ and the properties of ordinary non-supersymmetric YM. I will then show a somewhat more qualitative connection between SQCD and ordinary QCD.

\subsection{Linkage of $Y M$ to $\mathcal{N}=4 S Y M$}

Consider the linkage diagram in Fig. 9. We begin at the top with $\mathcal{N}=4$ $S U(N)$ SYM theory, a conformal field theory with a gauge coupling $g$. Under Olive-Montonen duality, this theory is rewritten, using magnetic variables, as $\mathcal{N}=4 S U(N) / \mathbf{Z}_{N}$ SYM, with gauge coupling $\tilde{g} \propto 1 / g$. Next, as discussed earlier, we take $g \gg 1$ and break $\mathcal{N}=4$ supersymmetry to $\mathcal{N}=1$ by adding finite masses $\mu$ for some of the fields. The resulting theory is strongly coupled, confines, and has chiral symmetry breaking, as is easily seen using the weaklycoupled magnetic variables, where the effect of the supersymmetry breaking is to cause the light monopoles to condense, breaking the gauge group completely, 


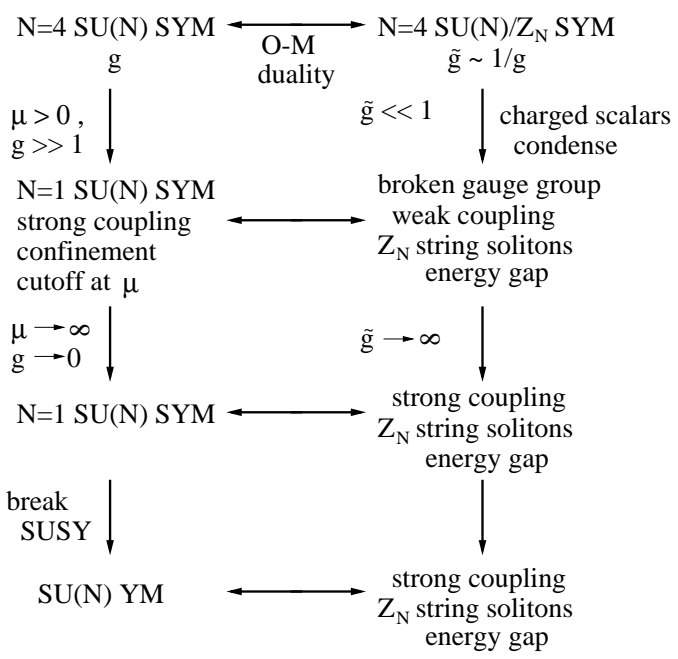

Figure 9: The connection of Olive-Montonen duality to confinement in Yang-Mills theory.

and leading to $\mathbf{Z}_{N}$-carrying solitonic flux tubes.

The following step is to take $g$ small and $\mu$ to infinity, holding the strong coupling scale fixed. The theory becomes pure $\mathcal{N}=1 \mathrm{SYM}$ in this limit, and maintains confinement and chiral symmetry breaking since it remains in the same universality class. ${ }^{d}$ The magnetic variables become strongly coupled as $g \rightarrow 0$, but we expect the $\mathbf{Z}_{N}$ solitonic strings will survive, since their existence is a consequence of the topology of the $S U(N) / \mathbf{Z}_{N}$ gauge group.

The last step is to break $\mathcal{N}=1 \mathrm{SYM}$ to YM. Here I must assume that there are no sharp transitions between these two theories - an issue which can and should be addressed on the lattice - in order to make the linkage complete. However, because SYM and YM share many properties, such a conjecture is quite plausible. If the transition between these two theories is smooth, then confinement and the $\mathbf{Z}_{N}$ flux tubes will survive from one theory to the other.

In summary, modulo the conjecture that $\mathcal{N}=1 \mathrm{SYM}$ and YM are continuously connected, the specific structure of duality in $\mathcal{N}=4 \mathrm{SYM}$ theories is directly related to - perhaps even implies - the fact that YM is a confining theory with $\mathbf{Z}_{N}$ flux tubes.

${ }^{d}$ See the previous footnote. 


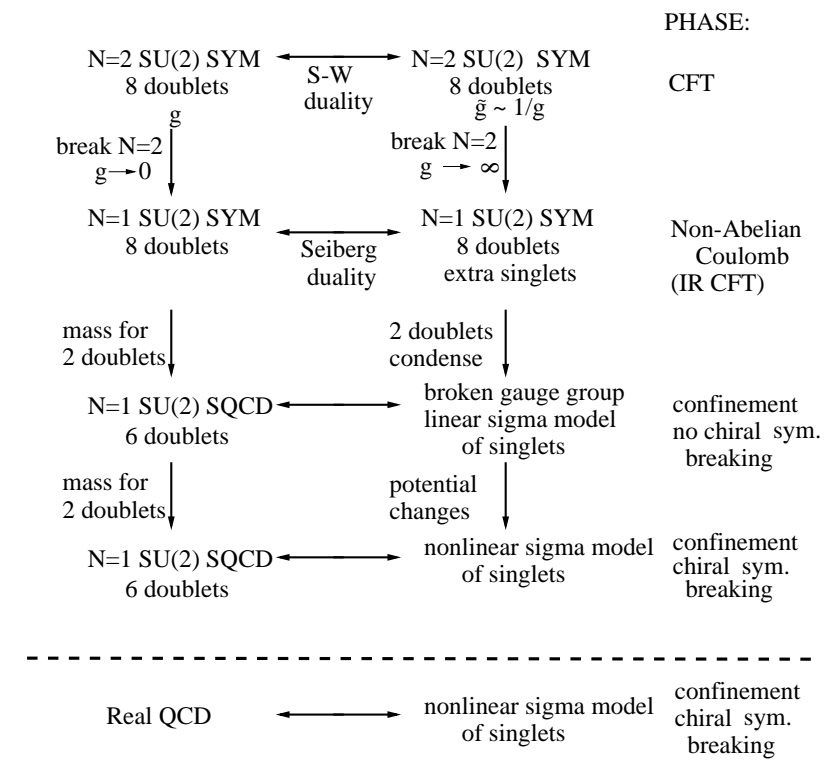

Figure 10: The connection of Seiberg-Witten and Seiberg duality to confinement, chiral symmetry breaking, and non-linear sigma models in QCD.

\subsection{Linkage of $Q C D$ to Duality in $\mathcal{N}=2, \mathcal{N}=1 S Q C D$}

Now I turn to my second linkage diagram, Fig. 10, which relates the duality of finite $\mathcal{N}=2$ theories to that of $\mathcal{N}=1$ theories and then to the properties of real QCD.

At the top of the diagram, we have a finite - and therefore conformal $\mathcal{N}=2$ SQCD theory, with gauge group $S U(2)$, gauge coupling $g$, and eight quarks and squarks in the doublet representation. ${ }^{e}$ As in the case of $\mathcal{N}=4$ SYM discussed earlier, this theory has a representation in terms of magnetic variables as another $\mathcal{N}=2$ SQCD theory, which in this case has the same gauge and matter content as the electric variables but has coupling constant $1 / g$. This duality transformation was discovered by Seiberg and Witten in their famous paper of $19944^{30}$

Now let us break $\mathcal{N}=2$ supersymmetry to $\mathcal{N}=1$ by giving mass to the extra fields in the $\mathcal{N}=2$ gauge multiplet. The theory becomes $\mathcal{N}=1$ SQCD with gauge group $S U(2)$ and eight quarks and squarks in the doublet

${ }^{e}$ The choice of $S U(2)$ is for simplicity only; the same physics applies with slight modification for any $S U(N)$. 
representation. This theory has a running coupling, but flows to a conformal fixed point in the infrared - it is in the non-abelian coulomb phase. The breaking of $\mathcal{N}=2$ supersymmetry causes the magnetic theory to flow to an $\mathcal{N}=1$ SQCD theory with the same charged matter content but with extra gauge singlets and interactions, ${ }^{41}$ precisely those required by Seiberg's $\mathcal{N}=1$ duality transformation. ${ }^{10}$ In other words, the Seiberg-Witten duality transformation of the $\mathcal{N}=2$ theory flows to the Seiberg duality transformation of $\mathcal{N}=1$ SQCD.

Now add masses for two of the electric doublets, leaving a theory of $S U(2)$ with six doublets. This causes some magnetic squarks to condense, breaking the $S U(2)$ magnetic gauge symmetry and leaving a theory of massless gauge singlet fields $M$. These singlets are precisely the mesons of the electric variables. Since the magnetic gauge symmetry is broken, electric charge is confined. Thus, confinement proceeds through a non-abelian generalization of the dual Meissner effect, and the low-energy magnetic theory - an infrared-free non-renormalizable theory with a cutoff — is the sigma model describing the confined hadrons. Examination of the sigma model, in particular the potential energy $V(M)$, shows that the the theory has a vacuum in which chiral symmetry is unbroken.

Adding masses for two more doublets merely causes the potential $V(M)$ to change in such a way that there is no longer a chiral-symmetry-preserving vacuum. Thus, $S U(2)$ SQCD with four doublets confines and breaks chiral symmetry. Shifting to the true vacuum and renaming the fields as representing fluctuations around that vacuum, we may rewrite the theory as a non-linear sigma model of pions and their superpartners.

To go from here to real non-supersymmetric QCD is a bit more of a stretch than in the previous linkage diagram, because the removal of the scalar squarks from the theory is rather more delicate and much less well understood. Rather than raise those questions, I leave the last step in the diagram as more of a heuristic one. It is evident that the duality in $\mathcal{N}=1 S U(2)$ SQCD with four doublets, relating the gauge theory of gluons, quarks and their superpartners to a non-linear sigma model of pions, is remarkably similar to the transformation between real-world QCD and the chiral Lagrangian that we use to describe its infrared physics. Indeed it is completely justified to call this QCD-tohadron transformation "duality". As we have seen, the duality in confining SQCD can be derived from the Seiberg-Witten duality of a conformal $\mathcal{N}=2$ SQCD theory. Is QCD-pion duality likewise embedded in a chain of nonsupersymmetric duality transformations similar to those in the diagram?

We are unable at this time to answer this question, even for non-chiral theories like QCD, because almost nothing is known about non-supersymmetric 
gauge theories other than $S U(2)$ and $S U(3)$ YM and QCD with a small number of flavors. This is where lattice QCD comes in, as it is at the present time almost the only tool available for studying this issue.

\section{Orbifolds? Here?}

Orbifolds of $S U(N)$ SYM are potentially rich sources of new insights. To my knowledge the importance of these theories was emphasized only recently. ${ }^{42,43}$ At the end of this section I will illustrate this through yet another linkage diagram.

Orbifolds of supersymmetric gauge theories are common in the recent string theory literature. A certain "parent" gauge theory is chosen; the orbifold theory is simply given by throwing away all fields which are not invariant under a discrete subgroup of the gauge symmetry. The resulting theory has the remarkable property that at large $N$ its perturbation series is the same as the parent theory, up to some simple rescaling of $N^{44,45,46,47}$ This is because the planar graphs have a very simple property under this projection. ${ }^{48}$ However, it is not proven that the relation between orbifold and parent holds non-perturbatively in the gauge coupling. Note that one cannot argue that non-perturbative effects are simply suppressed in the large- $N$ limit, as was long ago suggested from the fact that instanton effects are suppressed. Such arguments are clearly wrong in the case of $\mathcal{N}=1 \mathrm{SYM}$, where the gluino condensate does not vanish at large $N$. In short, the "orbifold conjecture" the suggestion that orbifold and parent are related even non-perturbatively is a non-trivial one.

For supersymmetric theories, the parent-orbifold connection often leads to very interesting results. Non-supersymmetric orbifolds, however, are usually unnatural. Most supersymmetric theories have massless scalar fields, and their orbifolds do as well. For non-supersymmetric orbifolds, the scalar fields remain massless only to leading order in $1 / N$. At higher order their masses have the usual quadratic divergences, which although multiplied by $1 / N$ are still divergent! In other words, the limit in which the cutoff is taken to infinity does not commute with the infinite $N$ limit. Thus, all models of this type with large but finite $N$ are fine-tuned. (Interesting observations have been made concerning these theories nonetheless ${ }^{44,47}$ although to what end I am unsure.) The important exception, however, is for orbifolds of SYM, which like SYM have no scalar fields, and which have fermions that are strictly massless due to chiral symmetries. These theories are natural and exhibit many phenomena that we would like to study.

I will focus exclusively on the non-supersymmetric $\mathbf{Z}_{p}$ orbifolds of SYM. 
These have $p S U(N)$ gauge factors, which I will label $S U(N)_{1}, S U(N)_{2}$, etc. There are also $p$ Weyl fermions $\psi_{i}, i=1, \ldots, p$, cyclically charged: $\psi_{i}$ is charged under two gauge groups, as $\mathbf{N}$ under $S U(N)_{i}$ and as $\overline{\mathbf{N}}$ under $S U(N)_{i+1}$. Like SYM, these theories also have discrete $\mathbf{Z}_{2 N}$ global symmetries which can be broken by fermion condensates. They also have a $\mathbf{Z}_{N}$ center under which flux tubes can be charged. ${ }^{f}$

The orbifold conjecture applies only when all gauge coupling constants are precisely equal. However, let us consider these theories with arbitrary gauge couplings, understanding that the relation with the parent theory only holds quantitatively when the couplings are exactly equal. This allows us to do a qualitative analysis of the infrared behavior of the theory. ${ }^{49}$ In particular, if there is a hierarchy in the gauge couplings, one can see, from the known physics of QCD, that these theories exhibit a form of tumbling. Suppose $S U(N)_{2}$ has the largest of the couplings. It will be the first, then, to become strongly coupled, with all the other gauge groups acting as weakly-coupled spectators to its dynamics. From QCD with three light flavors, we expect $S U(N)_{2}$ will confine and induce a condensate for $\psi_{1} \psi_{2}$. Just as in QCD, this condensate breaks $S U(N)_{1} \times S U(N)_{3}$ down to a diagonal subgroup $S U(N)_{D}$; in the breaking, the pions of $\psi_{1} \psi_{2}$ are eaten by the broken vector bosons. The combination of confinement of $S U(N)_{2}$ and breaking of $S U(N)_{1} \times S U(N)_{3} \rightarrow$ $S U(N)_{D}$ leaves $p-2$ gauge factors with $p-2$ Weyl fermions cyclically charged as before. In short, the $\mathbf{Z}_{p}$ orbifold tumbles dynamically to the $\mathbf{Z}_{p-2}$ orbifold. The next stage of confinement and chiral symmetry breaking removes two more factors, and so on.

If $p$ is odd, then the endpoint of this tumbling is simply the $p=1$ case, which is just $\mathcal{N}=1 \mathrm{SYM}$. In this case the low-energy effective theory has supersymmetry, as an accidental symmetry! The final step in the dynamics will involve SYM physics: confinement with a breaking of the discrete chiral symmetry, leaving a set of $N$ vacua which can have domain walls between them. If there are no phase transitions between this regime and the regime with all gauge couplings equal, then the orbifold limit will have the same properties. Thus its seems that the non-perturbative dynamics of the $S U(N)^{p}$ theory may indeed closely resemble those of an $S U(N)$ SYM theory. This make it seems likely - though difficult to verify - that when $N$ is large the relation between SYM and its orbifolds with $p$ odd goes well beyond perturbation theory in $g$.

Note also that the $p=3$ case is especially amusing. Take $N=3$; then our

${ }^{f}$ For the experts: I am ignoring the $U(1)$ factors that typically would arise in an orbifold. These are infrared free and cannot affect the non-perturbative physics in the infrared. If necessary they can easily be accounted for using perturbation theory, as with QED when discussing its effects on QCD; but they represent a $1 / N$ effect in any case. 
theory is QCD with three massless quarks, with the flavor symmetry $S U(3)_{L} \times$ $S U(3)_{R}$ gauged and with an additional fermion $\chi$ in the $(3, \overline{3})$ of $S U(3)_{L} \times$ $S U(3)_{R}$ to cancel gauge anomalies. The confinement and chiral symmetry breaking of ordinary QCD would make $\chi$ into an 8 (plus a decoupled singlet) of the remaining $S U(3)$ diagonal flavor symmetry - and thus into an effective gluino of the low-energy theory.

What if $p$ is even? Then the endpoint of the tumbling is the $\mathbf{Z}_{2}$ orbifold of SYM, which is especially interesting. It contains $S U(N) \times S U(N)$ with a Dirac fermion $\psi$ in the $(\mathbf{N}, \overline{\mathbf{N}})$ representation. Note, however, that the mass of the fermion must be zero, because of a $\mathbf{Z}_{N}$ discrete axial symmetry. For $N=3$, this is just QCD with three light quarks, with the diagonal subgroup of the chiral $S U(3)_{L} \times S U(3)_{R}$ flavor symmetry gauged. Thus, we expect this theory to have confinement and chiral symmetry breaking also, at least when one of the couplings is taken very small. Note that unlike QCD, however, the second $S U(N)$ ensures that there really is confinement here, with flux tubes that do not break and carry a $\mathbf{Z}_{N}$ charge. For example, sources in the $(\mathbf{N}, \mathbf{1})$ representation cannot be screened by the light matter. Also, instead of a continuous global symmetry and pions, as in QCD, the second $S U(N)$ breaks the global symmetry explicitly, making the pions pseudo-Goldstones. The only remaining symmetry is discrete, and we expect it to be broken by a $\bar{\psi} \psi$ condensate, giving $N$ degenerate vacua. Thus, like SYM, it will have domain walls.

The implications of this are exciting. What makes this orbifold theory special is that it can be simulated on the lattice with relative ease.

1) It can be studied when one of its gauge couplings is much larger than the other. In this case it should look like QCD with $S U(N)$-diagonal gauged, with the pions as a light charged adjoint, and with a secondary, low-energy confinement scale with associated flux tubes.

2) It can be studied when the gauge couplings are nearly equal. In this case, according to the orbifold proposal, many of its properties should look very similar to SYM. In the limit where the couplings are exactly equal and $N$ is taken moderately large, it should become quantitatively similar to SYM. For instance, the hadron spectrum should have surprising degeneracies. Furthermore, the relative tensions of domain walls should agree with those of SYM, where the domain walls are believed to be BPS-saturated and the ratios of their tensions are therefore exactly predicted! These relations allow a quantitative test of the orbifold conjecture beyond perturbation theory. Furthermore, if the orbifold conjecture is confirmed, this theory gives quantitative tests of predictions in $\mathcal{N}=1 S Y M$, even without simulating $S Y M$ on the lattice. It could therefore be used to resolve some of the puzzles still surrounding non- 


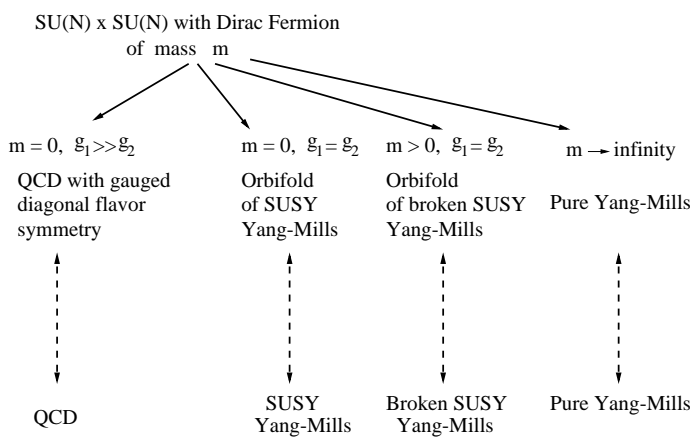

Figure 11: The $\mathbf{Z}_{2}$ orbifold of $\mathcal{N}=1$ SYM may be useful for the study of a number of interesting questions, as it has qualitative and quantitative connections with several important theories.

perturbative aspects of SYM.

3) Finally, the orbifold conjecture should survive quantitatively even when one compares broken $\mathcal{N}=1$ supersymmetry (with a mass for the gluino) to this $S U(N) \times S U(N)$ theory with a mass for the Dirac fermion. Thus, the $\mathbf{Z}_{2}$ orbifold is potentially a rich opportunity for studying various nonperturbative phenomena of QCD, SYM and YM simultaneously, through a theory which essentially is expected to interpolate between them. At worst, one will disprove the orbifold conjecture at the non-perturbative level; at best, one will both verify it and put it to quantitative use!

The interesting properties of the $\mathbf{Z}_{2}$ orbifold of $\mathcal{N}=1$ SYM are summarized in a third linkage diagram, given in Fig. 11.

\section{Large $N_{c}$ Gauge Theory and String Theory}

Now I want to turn to some even more spectacular developments of the last couple of years. I will be brief, but I hope to convey some of the key ideas nonetheless.

The fact that gauge theory, in the limit of a large number of colors, is in some way connected with string theory, with $1 / N$ playing the role of the string coupling, was first noted twenty-five years ago by 't Hooft. While many have attempted to make progress in understanding gauge theories by studying this limit further, a quantitative approach to $S U(N)$ YM or QCD using $1 / N$ as an expansion parameter has been stymied by the difficulty of determining the classical string theory which should appear in the $N \rightarrow \infty$ limit.

Let us consider some obvious facts about this string theory, and the gauge 
theories which might be studied using this approach. First, critical (that is, $d=10$ ) superstrings have gravity and supersymmetry. Since QCD has neither a spin-two hadron with only two polarization states (a graviton) nor supersymmetry, clearly its string theory is unrelated to critical superstrings. A second obvious fact is that only confining theories have physical string-like flux tubes and associated area laws for Wilson loops. Other theories, including conformal field theories such as $\mathcal{N}=4 \mathrm{SYM}$, have no physical string-like behavior and should not be associated with string theories.

We will not get anywhere using these obvious facts, however, because they are wrong. Following on the work of Gubser and Klebanov, ${ }^{11}$ and followed in turn by work of those authors with Polyakov, ${ }^{13}$ of Witten, ${ }^{14}$ and then of a flood of others, Maldacena proposed a bold conjecture ${ }^{12}$ relating supersymmetric conformal field theories in four dimensions to superstring theory. I will now state this conjecture, defining my terms as I go.

\subsection{Maldacena's Conjecture}

According to Maldacena's idea, $\mathcal{N}=4$ SYM theory with $N$ colors and coupling $g$ is related to Type IIB superstring theory (a ten-dimensional theory of closed strings with IIB supergravity as its low-energy limits - its massless fields are a graviton $G_{\mu \nu}$, antisymmetric tensor $B_{\mu \nu}$ and dilaton $\phi$ along with "Ramond-Ramond" 0 -index, 2-index and 4-index antisymmetric tensor fields $\chi, A_{\mu \nu}, C_{\mu \nu \rho \sigma}$.) The string theory exists on a ten-dimensional space consisting of a five-sphere $\left(x_{1}^{2}+x_{2}^{2}+x_{3}^{2}+x_{4}^{2}+x_{5}^{2}+x_{6}^{2}=R^{2}\right.$, a space of constant positive curvature) times a five-dimensional Anti de Sitter space $\left(-x_{1}^{2}-x_{2}^{2}+x_{3}^{2}+x_{4}^{2}+x_{5}^{2}+x_{6}^{2}=R^{2}\right.$, a space of constant negative curvature) with non-zero flux of $\partial_{\kappa} C_{\mu \nu \rho \sigma}$. The radius of the sphere and of the AdS space are both $R \propto\left(g^{2} N\right)^{1 / 4}$, so the curvature of the space is small at large $g^{2} N$. The string coupling $g_{s}$ is the square of the gauge coupling $g_{s} \propto g^{2} \propto R^{4} / N$. (Notice the $N$ dependence accords with 't Hooft's original observation.)

Where is the four-dimensional gauge theory in this ten-dimensional string? The five-dimensional AdS space has a four-dimensional boundary, and it is there that the gauge theory is to be found. Note that it has long been understood from the work of Polyakov that non-critical strings dynamically grow an extra dimension, so the presence of a five-(plus-five)-dimensional string theory in the context of a four-dimensional gauge theory is perhaps not so shocking. What is astonishing is that the string theory involved is the well-understood critical superstring, and that it is believed to be equivalent to the gauge theory on the boundary.

This conjecture has been extremely well-tested in the large $N$, large $g^{2} N$ 


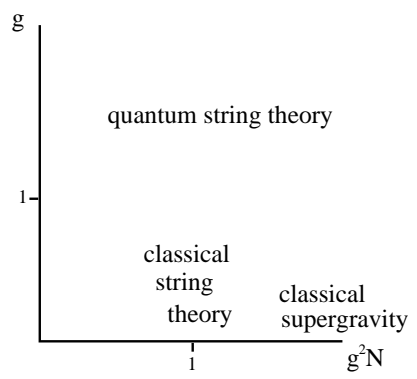

Figure 12: The conjectured duality between superstring theory and gauge theory connects gauge theory to classical superstring theory at small $g$ and further to classical supergravity at large $g^{2} N$.

regime, in which the full quantum string theory reduces to classical (small $g_{s}$ ) supergravity (small curvature), as shown in Fig. 12. The symmetries and operators of the two theories match; ${ }^{12}$ and the baryon operator (which is non-perturbative in string theory since $N \sim 1 / g_{s}$ ) has been identified with a D-brane (a soliton).$^{50}$ Furthermore, the Wilson loop of the gauge theory has been identified with the boundary of a string worldsheet in the larger space. ${ }^{51,52}$ This makes it possible to explain how this conformal $\mathcal{N}=4$ SYM theory can be stringy: although the Wilson loop is the boundary of a string, the string does not live on the boundary but hangs into the bulk, and so the value of the Wilson loop as a function of its size depends on the geometry outside the boundary. The difference between area law and perimeter law in various gauge theories thus is translated into differing spatial geometries in their string-theory duals.

These ideas have been further extended in a number of directions. In particular, it is easy to study finite temperature, and to use the fact that hightemperature five-dimensional SYM has infrared behavior equivalent to fourdimensional non-supersymmetric YM at strong coupling. ${ }^{54,55,56}$ It is straightforward to show that this strongly coupled theory confines. It is also possible to compute its spectrum of glueballs ${ }^{53}$ Remarkably, the ratios of certain glueball masses match rather well to lattice results in ordinary QCD away from strong coupling, both in three and four dimensions. However, although this is surprising and possibly an interesting statement about this particular strongcoupling limit, there is no clear reason for great excitement. There are many extra states in the spectrum which do not arise in YM theory; the glueball mass is not naturally related to the string tension; and there is no systematic approach toward recovering real YM theory starting from this limit. 
Other extensions include adding matter (which leads to both open and closed strings), reducing supersymmetry, changing the number of dimensions, and studying non-conformal theories at zero and finite temperature. Various expected phase transitions, such as deconfinement at high temperature, have been observed.

Recent work ${ }^{42,57,58,59}$ has brought us much closer to a study of $\mathcal{N}=1$ SYM, its QCD-like orbifold, and pure YM. These papers have shown several different methods for obtaining gravitational descriptions of theories in the same universality class as SYM. For example, one of these ${ }^{42}$ gives the stringy description of the breaking of $\mathcal{N}=4 \mathrm{SYM}$, as discussed earlier and illustrated in Fig. 9. All the properties of SYM - confinement, flux tubes, domain walls, baryon vertices, chiral symmetry breaking - have been identified as coming from geometry and from various branes and strings in various interesting topological or dynamical configurations. It is truly remarkable how the physics of strings and branes in a particular ten-dimensional spacetime manages to reproduce so much of the physics of SYM, and, when heavy quarks are added, of a theory resembling QCD. But this is an article in and of itself. I leave this topic for the future.

It should still be noted, however, serious obstacles lie in the path of any attempt to apply these string theory techniques to true SYM, ordinary YM or physical QCD, where for any fixed $N$ the value of $g$ runs such that $g^{2} N$ is not always large and $g$ is not always small. Where $g^{2} N$ is small, string theory is not a good description of the theory; perturbative field theory is, naturally, the only good one. In the regime where $g^{2} N$ is of order one, where the hadrons with mass of order $1 \mathrm{GeV}$ are likely to be found, supergravity is insufficient; string theory is required for the large $N$ limit. Unfortunately, almost nothing is known about string theory on an AdS background with Ramond-Ramond fields, even at the classical level, since the usual world-sheet formulation of string theory cannot be easily generalized to this case. Even were this problem solved, there is no guarantee that the solution will be easy to use. And worse, one must somehow understand quantitatively the transition from the perturbative gauge theory regime to the stringy regime. I cannot tell you whether these obstacles will be overcome tomorrow, next year, or in the fourth millennium; but in any case the difficulties are such that it seems unlikely these approaches will become a quantitative competitor to lattice gauge theory in the near future.

Nonetheless, it is remarkable that a sensible and definite proposal for the large $N$ expansion of gauge theory has been made and has passed some nontrivial tests - and that it appears to involve superstring theory! And we must certainly ask whether in fact superstring theory can be defined using gauge theory. 


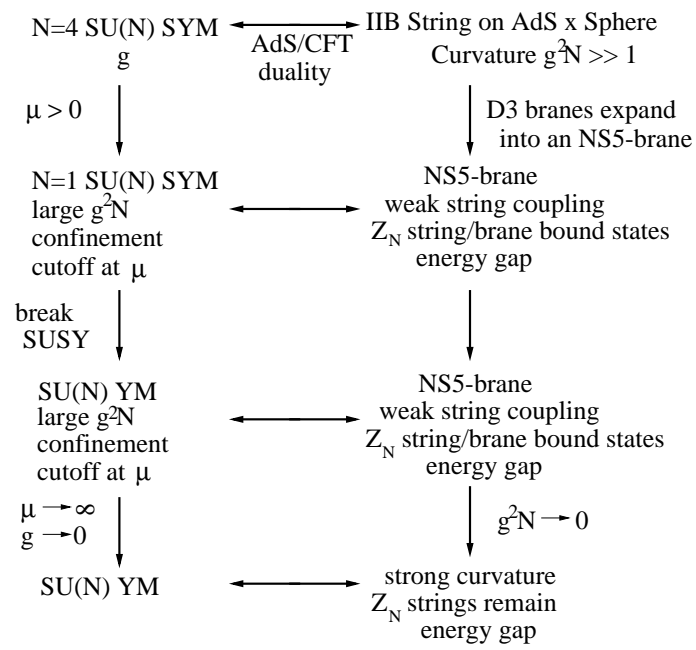

Figure 13: The Maldacena conjecture generalized to the case of $\mathcal{N}=4 \mathrm{SYM}$ broken to $\mathcal{N}=1 \mathrm{SYM}$, as in Fig. 9.

\subsection{A Final Linkage Diagram}

To again bring home how apparently esoteric results on theories with extended supersymmetry can have implications for real-world physics, I want to restate using Fig. 13 the connection between QCD and string theory as presently conjectured and partially understood. I must warn the reader that I will be speak rather loosely when describing this diagram, as this discussion is intended for novices who want only a rough idea of the physics. I ask experts to forgive the obvious misstatements.

Let us begin with $\mathcal{N}=4 \mathrm{SYM}$ and break it to $\mathcal{N}=1$, as in linkage diagram Fig. 9. According to the Maldacena conjecture this theory is dual to a superstring theory which is approximately $A d S_{5} \times S^{5}$ near the boundary of $A d S$ space, but which has an additional non-zero flux on it corresponding to the $\mathcal{N}=4$-breaking masses. As we move away from the boundary, this new flux grows and begins to react back on the spacetime. This back-reaction causes a Neveu-Schwarz (NS) 5-dimensional "brane" (an object which is dual to strings just as monopoles are dual to electrons) to appear out of the vacuum. ${ }^{42}$ It wraps around an $S^{2}$ of the $S^{5}$, as well as filling ordinary three-dimensional space. It can be shown that fundamental strings stretched along a direction of ordinary three-dimensional space can bind to this NS 5-brane. These strings correspond to electric flux in the gauge theory. The fact that they bind to the 
NS 5-brane, forming a state of definite tension, corresponds to the fact that in the SYM theory the flux lines cannot expand to arbitrarily low density, but instead make flux tubes of definite tension. One can also show these stringbrane bound states carry a $\mathbf{Z}_{N}$ charge. Indeed, the physics of Fig. 9 has a direct representation in the string theory.

\section{Outlook for QCD}

I will conclude with a short list of important questions raised in this article, and mention some specific ideas for future numerical work.

\subsection{Some questions}

First and foremost, what is duality? We still have no explicit understanding of non-trivial duality transformations in three or four dimensions, and not nearly enough even in two dimensions. Do we need a reformulation of field theory itself? What does duality in string theory teach us?

Does Olive-Montonen duality imply confinement in non-supersymmetric Yang-Mills theory by $\mathbf{Z}_{N}$-carrying electric flux tubes? I have shown that it does so for $\mathcal{N}=1 \mathrm{SYM}$, but one must show there is no phase boundary separating the two theories. What are the $\mathbf{Z}_{N}$ string tensions in pure $S U(N)$ YM? Do the ratios of tensions fit any known formulas, such as those of Eq. (18) or Eq. (19)?

What is the phase structure of QCD as a function of gauge group, matter content, and interactions? Does QCD have duality similar to SQCD? Is there a free magnetic phase?

Is the orbifold conjecture for large- $N$ field theory correct? Can it be verified, using lattice computations, for the QCD-like orbifold of $\mathcal{N}=1 \mathrm{SYM}$ described above? If it works, how can we best put it to use? Can the domain walls in the orbifold be quantitatively compared to those predicted in $\mathcal{N}=1$ SYM?

Can string theory give us a quantitative understanding of $\mathcal{N}=1$ SYM, and perhaps non-supersymmetric theories as well? Can it at least give us the hadron spectrum? Is the large- $N$ stringy expansion quantitatively tractable?

We have seen repeatedly that massive matter, when added to a theory, can make aspects of its physics easier to understand. What matter (or additional interactions) might we add to non-supersymmetric QCD to make some of these questions more accessible either analytically or numerically? 


\subsection{Some proposals for the lattice}

Within this set of questions, there are several projects that I hope will eventually be undertaken by the lattice community.

1) The transition from SQCD to QCD: I hope that it will soon be realistic to simulate $S U(2)$ or $S U(3)$ QCD with a Majorana spinor, of mass $m_{\lambda}$, in the adjoint representation. ${ }^{60}$ Tuning the mass to zero to make the theory $\mathcal{N}=1$ SQCD may be difficult, but many properties of the theory, including its strings, should not be sensitive to $m_{\lambda}$ as long as it is much smaller than the confinement scale $\Lambda$. It would be very interesting to map out the behavior of the theory, including the strings and their tensions $T_{k}$, as a function of $m_{\lambda} / \Lambda$. As I mentioned earlier, the absence of a transition in the properties of the strings would establish the linkage I have proposed between Olive-Montonen duality and the $\mathbf{Z}_{N}$ strings of $S U(N)$ YM.

2) Ratios of string tensions of YM: I have argued here that YM should be a type I superconductor, and that the same should hold for $\mathcal{N}=1 \mathrm{SYM}$ and for broken SYM. This conjecture must be studied in theories with at least four colors. It has passed its first test ${ }^{40}$ but there is much more to do before it can be conclusively verified.

3) $S U(N) \times S U(N)$ with a Dirac fermion in the $(\mathbf{N}, \overline{\mathbf{N}})$ : this $\mathbf{Z}_{2}$ orbifold of $\mathcal{N}=1$ SYM should be quantitatively similar to $\mathcal{N}=1$ SYM in many respects when $N$ is large. It would be interesting to study this theory on the lattice, at least for $N=2,3$ and 4; with the two gauge couplings equal, the trend toward a hadron spectrum with surprising degeneracies should be visible, and the ratio of the domain wall tensions in $S U(4)$ should be close to that predicted by $S U(4) \mathcal{N}=1 \mathrm{SYM}$. As emphasized in Fig. 11, there are several regions of interest where the theory has relations with pure QCD, pure YM, and broken $\mathcal{N}=1$ SYM.

4) Phase structure of QCD: A more difficult goal is to fully map out the low-energy phases of non-supersymmetric QCD as a function of gauge group, matter content, and interactions (including non-renormalizable ones.) In supersymmetric theories, we have begun to understand the complicated subject of the long distance physics of gauge theories as a function of their gauge group $G$, their matter representations $R_{i}$, and their interaction Lagrangian (including non-renormalizable terms.) Similar information would be welcome in the non-supersymmetric case. We know that the non-abelian coulomb phase exists at large $N_{c}, N_{f}$ when the one-loop beta function is very small, but how far does it extend away from this regime? What properties does the theory exhibit as it makes the transition from the perturbative regime to the conformal regime? The confining phase in supersymmetric theories is the exception, not the rule; 
which non-supersymmetric theories actually confine? Which ones break chiral symmetry, and in what patterns? What are their confining string tensions $T_{k}$ and their low-lying hadron spectra? Effects involving instantons, fractional instantons, monopoles, etc. may play an important role in some theories but which ones, and what effects are they responsible for? The free magnetic phase may not exist in non-supersymmetric theories - perhaps it requires the massless scalars of SQCD - but it would be very exciting if it were found (and a tremendous coup for the group which demonstrates its existence!) The existence of this phase would be a sufficient but not necessary condition for gauge theory-gauge theory duality in the non-supersymmetric context, which could also perhaps be shown in the context of the non-abelian coulomb phase. And of course, we must not leave out the possibility of new exotic effects which do not occur in the supersymmetric case.

It is important to emphasize that these questions are by no means of purely academic interest. The problem of electroweak symmetry breaking has not been solved, and there remains the possibility that the symmetry breaking occurs through a technicolor-like scenario, in which it is driven by chiral symmetry breaking in a strongly-coupled gauge theory. Technicolor models with physics similar to QCD have been ruled out by precision measurements at LEP. However, if the physics is considerably different from QCD, then we have no predictive tools, and therefore no experimental constraints. It will be an embarrassment to theorists if the LHC discovers evidence of strong dynamics of a type that we simply do not recognize. It is therefore important for model-building and for comparison with experiment that we improve our understanding of strongly-coupled non-supersymmetric theories.

I should mention also that there are potential spinoffs from such a program in the areas of supersymmetry breaking (which can be detected but not quantitatively studied using presently available analytic techniques) and in condensed matter systems where many similar physics issues arise.

To answer these questions, which require studying theories with very light fermions and often a hierarchy of physical scales, will require powerful lattice techniques not yet fully developed. I encourage the reader to think about how best to pursue this program of study! ${ }^{g}$

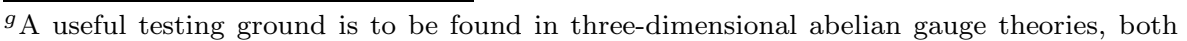
with and without supersymmetry. The supersymmetric theories are known to have an intricate phase structure, with a duality transformation (called "mirror symmetry") 61,31 and interesting large $N_{f}$ behavior. There are examples of non-trivial fixed points, infrared-free mirror gauge theories, chiral symmetry breaking and confinement. Many of these phases are likely to show up in the non-supersymmetric case, and lattice approaches to studying non-supersymmetric QCD could be tested in these theories. 


\section{Acknowledgements}

I am grateful to many colleagues for conversations, including M. Alford, D. Kabat, R. Leigh, A. Hanany, K. Intriligator, I. Klebanov, J. March-Russell, J. Polchinksi, N. Seiberg, F. Wilczek, and E. Witten. I am also grateful to M. Shifman for asking me to write this review, and to T. De Grand and T. Suzuki for encouraging me to push these ideas forward. This work was supported by the National Science Foundation under Grant PHY-9513835 and by the WM Keck Foundation, and by Department of Energy grant DE-FG02-95ER40893.

\section{References}

1. K. Intriligator and N. Seiberg, in Nucl. Phys. Proc. Suppl. 45BC, 1 (1996) hep-th/9509066.

2. A. Bilal, hep-th/9601007.

3. M. Chaichian, W. F. Chen and C. Montonen, hep-th/0007240.

4. O. Aharony, S. S. Gubser, J. Maldacena, H. Ooguri and Y. Oz, Phys. Rept. 323, 183 (2000) hep-th/9905111.

5. T. Appelquist, J. Terning and L. C. Wijewardhana, Phys. Rev. Lett.79, 2767 (1997), hep-ph/9706238.

6. N. Seiberg, Phys. Rev. D49, 6857 (1994) hep-th/9402044.

7. N. Seiberg, hep-th/9408013.

8. N. Seiberg and E. Witten, Nucl. Phys. B 426, 19 (1994), hepth/9407087, Erratum-ibid. B430 (1994) 485.

9. M.R. Douglas and S.H. Shenker, Nucl. Phys. B 47, 271 (1995), hepth/9503163.

10. N. Seiberg, Nucl. Phys. B 435, 129 (1995), hep-th/9411149.

11. S. S. Gubser and I. R. Klebanov, Phys. Lett. B413, 41 (1997), hepth/9708005.

12. J. Maldacena, Adv. Theor. Math. Phys. 2, 231 (1998) hep-th/9711200.

13. S. S. Gubser, I. R. Klebanov and A. M. Polyakov, Phys. Lett. B428, 105 (1998) hep-th/9802109.

14. E. Witten, Adv. Theor. Math. Phys. 2, 253 (1998), hep-th/9802150

15. K. Intriligator and N. Seiberg, Nucl. Phys. B 444, 125 (1995), hepth/9503179.

16. K. Intriligator and N. Seiberg, in STRINGS 95: Future Perspectives in String Theory, Los Angeles, CA, hep-th/9506084.

17. D. Kutasov, Phys. Lett. B 351, 230 (1995), hep-th/9503086; D. Kutasov and A. Schwimmer, Phys. Lett. B 354, 315 (1995), hep-th/9505004.

18. R.G. Leigh and M.J. Strassler, Nucl. Phys. B 447, 95 (1995), hepth/9503121; K. Intriligator and P. Pouliot, Phys. Lett. B 353, 471 
(1995), hep-th/9505006; K. Intriligator, R.G. Leigh and M.J. Strassler, Nucl. Phys. B 456, 567 (1995), hep-th/9506148.

19. P. Pouliot, Phys. Lett. B 359, 108 (1995)hep-th/9507018.

20. T. Banks and A. Zaks, Nucl. Phys. B 196, 189 (1982) .

21. M. J. Strassler, J. High Energy Phys.9809, 017 (1998), hep-th/9709081.

22. M.J. Strassler, talks given at YKIS '97, Kyoto, Japan, Minneapolis, MN, hep-th/9808073.

23. M.J. Strassler, talk given at QCD '98, Minneapolis, MN, hep-th/9808073.

24. E. Fradkin and S.H. Shenker, Phys. Rev. D 19, 3682 (1979); T. Banks and A. Casher, Nucl. Phys. B 169, 103 (1980).

25. See, for example, D. Anselmi, J. Erlich, D.Z. Freedman and A.A. Johansen, hep-th/9711035.

26. C. Montonen and D. Olive, Phys. Lett. B 72, 117 (1977).

27. P. Goddard, J. Nuyts and D. Olive, Nucl. Phys. B 125, 1 (1977).

28. H. Osborn, Phys. Lett. B 83, 321 (1979).

29. R. Donagi and E. Witten, Nucl. Phys. B 460, 299 (1996), hepth/9510101.

30. N. Seiberg and E. Witten, Nucl. Phys. B 431, 484 (1994), hepth/9408099.

31. A. Kapustin and M. J. Strassler, J. High Energy Phys.9904, 021 (1999), hep-th/9902033.

32. K. Hori and C. Vafa, hep-th/0002222.

33. C. Vafa and E. Witten, Nucl. Phys. B 431, 3 (1994), hep-th/9408074.

34. A. Klemm, W. Lerche and S. Yankielowicz and S. Theisen, Phys. Lett. B 344, 169 (1995), hep-th/9411048; P.C. Argyres and A.E. Faraggi, Phys. Rev. Lett. 74, 3931 (1995), hep-th/9411057; A. Hanany and Y. Oz, Nucl. Phys. B 452, 283 (1995), hep-th/9505075.

35. A. Hanany, M. J. Strassler and A. Zaffaroni, Nucl. Phys. B513, 87 (1998), hep-th/9707244.

36. E. Witten, Phys. Lett. B 86, 283 (1979).

37. E. Witten, Nucl. Phys. B 202, 253 (1982) .

38. E. Witten, Nucl. Phys. B 507, 658 (1997), hep-th/9706109.

39. N. Evans and M. Schwetz, hep-th/9708122.

40. S. Ohta and M. Wingate, in NONE Nucl. Phys. Proc. Suppl. 73, 435 (1999), hep-lat/9808022.

41. R. G. Leigh and M. J. Strassler, Nucl. Phys. B447, 95 (1995), hepth/9503121.

42. J. Polchinski and M. J. Strassler, hep-th/0003136.

43. M. J. Strassler, in preparation.

44. S. Kachru and E. Silverstein, Phys. Rev. Lett. 80, 4855 (1998) hep- 
th/9802183.

45. A. Lawrence, N. Nekrasov and C. Vafa, Nucl. Phys. B533, 199 (1998), hep-th/9803015.

46. M. Bershadsky, Z. Kakushadze and C. Vafa, Nucl. Phys. B523, 59 (1998), hep-th/9803076.

47. M. Schmaltz, Phys. Rev. D59, 105018 (1999) hep-th/9805218.

48. M. Bershadsky and A. Johansen, Nucl. Phys. B536, 141 (1998), hepth/9803249.

49. H. Georgi, Nucl. Phys. B266, 274 (1986).

50. E. Witten, J. High Energy Phys.9807, 006 (1998) hep-th/9805112.

51. J. Maldacena, Phys. Rev. Lett.80, 4859 (1998), hep-th/9803002.

52. S. Rey and J. Yee, hep-th/9803001.

53. C. Csaki, H. Ooguri, Y. Oz and J. Terning, J. High Energy Phys.9901, 017 (1999), hep-th/9806021.

54. E. Witten, Adv. Theor. Math. Phys. 2, 505 (1998), hep-th/9803131.

55. S. Rey, S. Theisen and J. Yee, Nucl. Phys. B527, 171 (1998), hepth/9803135.

56. A. Brandhuber, N. Itzhaki, J. Sonnenschein and S. Yankielowicz, Phys. Lett. B434, 36 (1998), hep-th/9803137.

57. I. R. Klebanov and M. J. Strassler, J. High Energy Phys.0008, 052 (2000), hep-th/0007191.

58. J. M. Maldacena and C. Nunez, hep-th/0008001.

59. C. Vafa, hep-th/0008142.

60. I. Montvay, Nucl. Phys. B 466, 259 (1996), hep-lat/9510042; heplat/9709080; N. Evans, S.D.H. Hsu and M. Schwetz, hep-th/9707260; A. Donini, M. Guagnelli, P. Hernandez and A. Vladikas, hep-lat/9710065.

61. K. Intriligator and N. Seiberg, Phys. Lett. B387, 513 (1996) hepth/9607207. 